TRANSACTIONS OF THE

AMERICAN MATHEMATICAL SOCIETY

Volume 358, Number 7, Pages 3077-3096

S 0002-9947(06)03928-6

Article electronically published on February 20, 2006

\title{
PROJECTIVE FRAÏSSÉ LIMITS AND THE PSEUDO-ARC
}

\author{
TREVOR IRWIN AND SŁAWOMIR SOLECKI
}

\begin{abstract}
The aim of the present work is to develop a dualization of the Fraïssé limit construction from model theory and to indicate its surprising connections with the pseudo-arc. As corollaries of general results on the dual Fraïssé limits, we obtain Mioduszewski's theorem on surjective universality of the pseudo-arc among chainable continua and a theorem on projective homogeneity of the pseudo-arc (which generalizes a result of Lewis and Smith on density of homeomorphisms of the pseudo-arc among surjective continuous maps from the pseudo-arc to itself). We also get a new characterization of the pseudo-arc via the projective homogeneity property.
\end{abstract}

\section{INTRODUCTION}

In the first part of the paper we dualize the classical (injective) Fraïssé limit found in model theory [5. The appropriate setting for it will be provided by topological $L$-structures, where $L$ is a language of relational and functional symbols and where by a topological $L$-structure $D$ we mean a compact, second countable, zero-dimensional space equipped with interpretations of relation symbols of $L$ as closed subsets of $D^{k}$ and of function symbols of $L$ as continuous functions from $D^{k}$ to $D$ for various $k \in \mathbb{N}$. Morphisms between such structures, which will be defined in the next section, are always continuous.

We consider countable families of finite topological $L$-structures equipped with the discrete topology which satisfy certain "refinement" properties. These refinement properties will be stated precisely later. Whereas in the classical theory of Fraïssé limits one considers injective homomorphisms, here we take projective homomorphisms. We will make this concept precise with the definition of what we will call an epimorphism. In Theorem 2.4 we show that if $\Delta$ is a class satisfying these refinement properties, then there exists a topological $L$-structure $\mathbb{D}$, the projective Fraïssé limit of $\Delta$, which is both projectively universal and projectively ultrahomogeneous with respect to $\Delta$. This means that every member of $\Delta$ is an epimorphic image of $\mathbb{D}$, and given any epimorphisms $\phi_{1}, \phi_{2}$ from $\mathbb{D}$ to some $D \in \Delta$ there is an isomorphism $\psi$ of $\mathbb{D}$ such that $\phi_{2}=\phi_{1} \circ \psi$. The topological $L$-structure $\mathbb{D}$ may be represented as an inverse limit of elements from $\Delta$. We show that $\mathbb{D}$ is unique up to isomorphism. If $\Delta$ is infinite, then $\mathbb{D}$ is a non-discrete compact space. Contrast this with the classical Fraïssé construction where one obtains countable Fraïssé limits with no topology on them.

Received by the editors July 7, 2004.

2000 Mathematics Subject Classification. Primary 03C98, 54F15.

Key words and phrases. Fraïssé limit, pseudo-arc.

The second author was partially supported by NSF grant DMS-0102254.

(C)2006 American Mathematical Society Reverts to public domain 28 years from publication 3077 
In the second part, with an eye towards the application that follows in the final section of the paper, we introduce the family $\Delta_{0}$ of finite linear (reflexive) graphs. We show in Theorem 3.1 that this class satisfies the refinement properties, and thus has a projective Fraïssé limit $\mathbb{P}$.

Finally in part three, we establish a connection between projective Fraïssé limits and the pseudo-arc. (For definitions related to continua and the pseudo-arc see the last paragraph of this section.) In Theorem 4.2 we show that by appropriately moding out the model theoretic content of $\mathbb{P}$, we obtain as a quotient space a hereditarily indecomposable chainable continuum, i.e., the pseudo-arc. By using the fact that the pseudo-arc is a quotient space of $\mathbb{P}$, we are able to transfer properties of $\mathbb{P}$ to the pseudo-arc. Thus with Theorem 4.4(i) we give a proof of Mioduszewski's universality theorem [9] that each chainable continuum is the continuous image of the pseudo-arc. In Theorem 4.4(ii) we establish a homogeneity result stating that for any two continuous surjections $f_{1}, f_{2}$ from the pseudo-arc onto the same chainable continuum there exists a homeomorphism $h$ of the pseudo-arc with $f_{2} \circ h$ as close to $f_{1}$ in the uniform topology as required. This extends a result of Lewis [7] and Smith [13]. Both these results are obtained as direct consequences of general properties of arbitrary projective Fraïssé limits. This indicates that the theorem of Lewis and Smith can be viewed as a homogeneity result for the pseudo-arc, and that the generalization of this result proved here and Mioduszewski's theorem can be seen as two phenomena (homogeneity and universality) linked at a deeper level. Informed by the analogy with projective Fraïssé limits, we show in Theorem 4.9 that the pseudo-arc is the unique chainable continuum fulfilling the conclusion of the homogeneity theorem. This gives a new characterization of the pseudo-arc.

Recall that a continuum is a compact connected metric space. For a compact metric space $X$, we say that an open cover $\mathcal{U}$ of $X$ refines an open cover $\mathcal{V}$ if each element of $\mathcal{U}$ is contained in an element of $\mathcal{V}$. We call a continuum $X$ chainable if each open cover of $X$ is refined by an open cover $U_{1}, \ldots, U_{n}$ such that for $i, j \leq n$, $U_{i} \cap U_{j} \neq \varnothing$ if and only if $|i-j| \leq 1$. Such a cover of $X$ is called a chain. A continuum is indecomposable if it is not the union of two proper subcontinua. A continuum is hereditarily indecomposable if each of its subcontinua is indecomposable. The pseudo-arc is the unique hereditarily indecomposable chainable continuum. It is also the generic continuum: in the (compact) space of all subcontinua of $[0,1]^{\mathbb{N}}$ equipped with the Hausdorff metric, homeomorphic copies of the pseudo-arc form a dense $G_{\delta}$ set. Readers interested in learning more about the pseudo-arc should see [8].

\section{The PRojective Fraïssé Limit}

2.1. Definition and elementary lemmas. Let $L$ be a language consisting of relation symbols $R_{i}, i \in I$, with arity $m_{i} \in \mathbb{N}$, and function symbols $f_{j}, j \in J$, with arity $n_{j} \in \mathbb{N}$. By a topological L-structure we mean a zero-dimensional, compact, second countable space $A$ together with closed sets $R_{i}^{A} \subseteq A^{m_{i}}$ and continuous functions $f_{j}^{A}: A^{n_{j}} \rightarrow A$ for all $i \in I$ and $j \in J$. Let $A$ and $B$ be two topological $L$-structures. By an epimorphism from $A$ to $B$ we mean a surjective continuous function $\phi: A \rightarrow B$ such that for any $j \in J$ and $x_{1}, \ldots, x_{n_{j}} \in A$ we have

$$
f_{j}^{B}\left(\phi\left(x_{1}\right), \ldots, \phi\left(x_{n_{j}}\right)\right)=\phi\left(f_{j}^{A}\left(x_{1}, \ldots, x_{n_{j}}\right)\right)
$$


and for any $i \in I$ and any $y_{1}, \ldots, y_{m_{i}} \in B$ we have

$$
\begin{aligned}
& \left(y_{1}, \ldots, y_{m_{i}}\right) \in R_{i}^{B} \\
& \Leftrightarrow \exists x_{1}, \ldots, x_{m_{i}} \in A\left(\phi\left(x_{p}\right)=y_{p} \text { for all } p \leq m_{i} \text { and }\left(x_{1}, \ldots, x_{m_{i}}\right) \in R_{i}^{A}\right) .
\end{aligned}
$$

By an isomorphism we mean a bijective epimorphism. Since the topology on a topological $L$-structure is compact, each isomorphism is a homeomorphism. Note also that if $\phi: A \rightarrow B$ is an isomorphism, (2.2) is equivalent to

$$
\left(\phi\left(x_{1}\right), \ldots, \phi\left(x_{m_{i}}\right)\right) \in R_{i}^{B} \Leftrightarrow\left(x_{1}, \ldots, x_{m_{i}}\right) \in R_{i}^{A} .
$$

We say that an epimorphism $\phi: A \rightarrow B$ between two topological $L$-structures $A$ and $B$ refines an open covering $\mathcal{U}$ of $A$ if for each $y \in B$ there is a $U \in \mathcal{U}$ with $\phi^{-1}(y) \subseteq U$.

The following lemma encodes a crucial property of epimorphisms. Since its proof is simple diagram chasing, we leave it to the reader.

Lemma 2.1. Let $A, B, C$ be topological L-structures. Let $f: B \rightarrow A, g: C \rightarrow A$, $\phi: C \rightarrow B$ be functions such that $g=f \circ \phi$. Assume that $\phi$ is an epimorphism. Then $f$ is an epimorphism iff $g$ is an epimorphism.

Let $\Delta$ be a family of topological $L$-structures. We say that $\Delta$ is a projective Fraissé family if the following two conditions hold:

(F1) for any $D, E \in \Delta$ there is an $F \in \Delta$ and epimorphisms from $F$ onto $D$ and onto $E$;

(F2) for any $C, D, E \in \Delta$ and any epimorphisms $\phi_{1}: D \rightarrow C, \phi_{2}: E \rightarrow C$, there exists an $F \in \Delta$ with epimorphisms $\psi_{1}: F \rightarrow D$ and $\psi_{2}: F \rightarrow E$ such that $\phi_{1} \circ \psi_{1}=\phi_{2} \circ \psi_{2}$.

Let $\Delta$ be a family of topological $L$-structures. We say that a topological $L$ structure $\mathbb{D}$ is a projective Fraissé limit of $\Delta$ if the following three conditions hold:

(L1) (projective universality) for any $D \in \Delta$ there is an epimorphism from $\mathbb{D}$ to $D$;

(L2) for any finite discrete topological space $A$ and any continuous function $f: \mathbb{D} \rightarrow A$ there is a $D \in \Delta$, an epimorphism $\phi: \mathbb{D} \rightarrow D$, and a function $f^{\prime}: D \rightarrow A$ such that $f=f^{\prime} \circ \phi$;

(L3) (projective ultrahomogeneity) for any $D \in \Delta$ and any epimorphisms $\phi_{1}$ : $\mathbb{D} \rightarrow D$ and $\phi_{2}: \mathbb{D} \rightarrow D$ there exists an isomorphism $\psi: \mathbb{D} \rightarrow \mathbb{D}$ such that $\phi_{2}=\phi_{1} \circ \psi$.

The conclusion of the following lemma gives a convenient restatement of (L2).

Lemma 2.2. Let $\Delta$ be a family of topological L-structures. Let $\mathbb{D}$ be a topological $L$ structure fulfilling (L2). Then for each open covering of $\mathbb{D}$ there is an epimorphism from $\mathbb{D}$ to a structure in $\Delta$ refining the covering.

Proof. Given an open cover of $\mathbb{D}$ find a finite cover $A$ consisting of clopen sets and refining it. Then define $f: \mathbb{D} \rightarrow A$ by letting $f(x)$ be the element of $A$ containing $x$. Now apply (L2).

Lemma 2.3. Let $\Delta$ be a projective Fraïssé family of finite topological L-structures and let $\mathbb{D}$ be a projective Fraïssé limit of $\Delta$. Let $D, E \in \Delta$ and let $\phi: E \rightarrow D$ and $\psi: \mathbb{D} \rightarrow D$ be epimorphisms. Then there exists an epimorphism $\chi: \mathbb{D} \rightarrow E$ such that $\phi \circ \chi=\psi$. 
Proof. By (L1) there is an epimorphism $\alpha: \mathbb{D} \rightarrow E$. We now have two epimorphisms $\psi$ and $\phi \circ \alpha$ from $\mathbb{D}$ to $D$. Thus, by (L3) there is an isomorphism $\beta: \mathbb{D} \rightarrow \mathbb{D}$ such that $(\phi \circ \alpha) \circ \beta=\psi$. Take $\chi$ to be $\alpha \circ \beta$.

2.2. Existence and uniqueness. In the following theorem we show that each countable projective Fraïssé family of finite topological $L$-structures has a projective Fraïssé limit which is unique.

Theorem 2.4. Let $\Delta$ be a countable projective Fraissé family of finite topological L-structures.

(i) There exists a topological L-structure which is a projective Fraïssé limit of $\Delta$.

(ii) Any two topological L-structures which are projective Fraïssé limits of $\Delta$ are isomorphic.

Proof. We prove (i) first. Inductively construct a sequence $\left(D_{n}\right)_{n \in \mathbb{N}}$ of structures in $\Delta$ and epimorphisms $\pi_{n}: D_{n+1} \rightarrow D_{n}$ so that the following conditions are satisfied where $\pi_{n}^{m}$, for $m>n$, stands for $\pi_{n} \circ \cdots \circ \pi_{m-1}: D_{m} \rightarrow D_{n}$ :

(a) for any $D \in \Delta$ there is an $n$ and an epimorphism from $D_{n}$ to $D$;

(b) for any $n$, any pair $E, F \in \Delta$, and any epimorphisms $\phi_{1}: F \rightarrow E$ and $\phi_{2}: D_{n} \rightarrow E$ there exists $m>n$ and an epimorphism $\psi: D_{m} \rightarrow F$ such that

$$
\phi_{1} \circ \psi=\phi_{2} \circ \pi_{n}^{m} .
$$

This construction is easy to carry out by recursion. We use the countability of $\Delta$ and achieve point (a) by applying (F1) and point (b) by applying (F2).

Let $\mathbb{D}=\lim \left(D_{n}, \pi_{n}\right)$. Let $\pi_{m}^{\infty}$ be the natural projection from $\mathbb{D}$ to $D_{m}$. If $R$ is a relation symbol in $L$ of arity $k$ and $x_{1}, \ldots, x_{k} \in \mathbb{D}$, we let $\left(x_{1}, \ldots, x_{k}\right) \in R^{\mathbb{D}}$ precisely when for all $n \in \mathbb{N},\left(\pi_{n}^{\infty}\left(x_{1}\right), \ldots, \pi_{n}^{\infty}\left(x_{k}\right)\right) \in R^{D_{n}}$. Similarly, if $f$ is a function symbol of arity $k$ and $x_{1}, \ldots, x_{k} \in \mathbb{D}$, we let $f^{\mathbb{D}}\left(x_{1}, \ldots, x_{k}\right)=y$ for the unique $y \in \mathbb{D}$ with $f^{D_{n}}\left(\pi_{n}^{\infty}\left(x_{1}\right), \ldots, \pi_{n}^{\infty}\left(x_{k}\right)\right)=\pi_{n}^{\infty}(y)$ for all $n$. Clearly $R^{\mathbb{D}}$ is closed and $f^{\mathbb{D}}$ is continuous.

We now check that $\mathbb{D}$ is a projective Fraïssé limit of $\Delta$.

Claim 1. $\pi_{m}^{\infty}$ is an epimorphism.

Proof of Claim 1. Let $R$ be a $k$-ary relation symbol. If $x_{1}, \ldots, x_{k} \in \mathbb{D}$ and we have $\left(x_{1}, \ldots, x_{k}\right) \in R^{\mathbb{D}}$, then by the definition of $R^{\mathbb{D}},\left(\pi_{m}^{\infty}\left(x_{1}\right), \ldots, \pi_{m}^{\infty}\left(x_{k}\right)\right) \in R^{D_{m}}$. Now let $d_{1}, \ldots, d_{k} \in D_{m}$ be such that $\left(d_{1}, \ldots, d_{k}\right) \in R^{D_{m}}$. We need to find $x_{1}, \ldots, x_{k} \in$ $\mathbb{D}$ such that $\left(x_{1}, \ldots, x_{k}\right) \in R^{\mathbb{D}}$ and $d_{i}=\pi_{m}^{\infty}\left(x_{i}\right)$ for $i \leq k$. Since each $\pi_{n}$ is an epimorphism, by recursion we choose $d_{1}^{n}, \ldots d_{k}^{n} \in D_{n}$ for $n \geq m$ so that for $i \leq k$

$$
d_{i}^{m}=d_{i}, \pi_{n}\left(d_{i}^{n+1}\right)=d_{i}^{n}, \text { and }\left(d_{1}^{n}, \ldots d_{k}^{n}\right) \in R^{D_{n}} .
$$

It follows now from the definition of $\mathbb{D}$ that there are $x_{1}, \ldots, x_{k} \in \mathbb{D}$ with $\pi_{n}^{\infty}\left(x_{i}\right)=$ $d_{i}^{n}$ for $n \geq m$ and $i \leq k$. In particular, we get $\pi_{m}^{\infty}\left(x_{i}\right)=d_{i}$ for $i \leq k$ and $\left(\pi_{n}^{\infty}\left(x_{1}\right), \ldots, \pi_{n}^{\infty}\left(x_{k}\right)\right) \in R^{D_{n}}$ for all $n \geq m$, and therefore for all $n$. The last formula implies that $\left(x_{1}, \ldots, x_{k}\right) \in R^{\mathbb{D}}$.

Similarly we check the condition concerning function symbols and the claim is proved.

Since $\pi_{m}^{\infty}$ maps $\mathbb{D}$ onto $D_{m}$, we get (L1) by Claim 1 and by point (a) from the construction. To see (L2), note that if $A$ is a finite space, then any continuous 
function from $\mathbb{D}$ to $A$ factors through $\pi_{n}^{\infty}: \mathbb{D} \rightarrow D_{n}$ for some $n$; thus, (L2) follows from Claim 1 as well.

It remains to prove (L3). First we show the following claim.

Claim 2. Let $\chi: D_{n} \rightarrow D_{k}, n>k$, be an epimorphism. There exists an isomorphism $\psi$ of $\mathbb{D}$ such that $\chi \circ \pi_{n}^{\infty}=\pi_{k}^{\infty} \circ \psi$.

Proof of Claim 2. We construct sequences $\left(k_{i}\right)$ and $\left(n_{i}\right)$ of natural numbers so that $k_{0}=k, n_{0}=n$, and $k_{i}<n_{i} \leq k_{i+1}$, for all $i$. We also construct epimorphisms $\chi_{i}: D_{n_{i}} \rightarrow D_{k_{i}}$ and $\alpha_{i}: D_{k_{i+1}} \rightarrow D_{n_{i}}$ so that $\chi_{0}=\chi$ and

$$
\chi_{i} \circ \alpha_{i}=\pi_{k_{i}}^{k_{i+1}} \text { and } \alpha_{i} \circ \chi_{i+1}=\pi_{n_{i}}^{n_{i+1}} .
$$

This is accomplished by induction. Set $\chi_{0}=\chi$. Assume we have $k_{i}, n_{i}$, and $\chi_{i}$. We will show how to get $k_{i+1}$ and $\alpha_{i}$. (One similarly produces $n_{i+1}$ and $\chi_{i+1}$.) We apply point (b) to the pair $D_{k_{i}}, D_{n_{i}}$, and the epimorphisms $\chi_{i}: D_{n_{i}} \rightarrow D_{k_{i}}$ and id : $D_{k_{i}} \rightarrow D_{k_{i}}$ to get an $m>k_{i}$ and an epimorphism $\psi: D_{m} \rightarrow D_{n_{i}}$ such that $\chi_{i} \circ \psi=\pi_{k_{i}}^{m}$. Note that $m \geq n_{i}$. Let $k_{i+1}=m$ and $\alpha_{i}=\psi$. Now, it follows immediately from (2.3) and the fact that $\chi_{0}=\chi$ that the sequence $\left(\chi_{i}\right)$ induces an isomorphism $\psi$ on $\mathbb{D}$ as required by the claim. Indeed, equations (2.3) show that the sequence $\left(\alpha_{i}\right)$ induces a function from $\mathbb{D}$ to itself which is both the left and right inverse of $\psi$. Thus, $\psi$ is a bijection. It is an epimorphism since each $\chi_{i}$ is an epimorphism.

To get (L3), let $\phi_{1}$ and $\phi_{2}$ be epimorphisms from $\mathbb{D}$ to some $D \in \Delta$. There are $n_{1}, n_{2} \in \mathbb{N}$ and functions $\phi_{1}^{\prime}: D_{n_{1}} \rightarrow D$ and $\phi_{2}^{\prime}: D_{n_{2}} \rightarrow D$ such that

$$
\phi_{1}=\phi_{1}^{\prime} \circ \pi_{n_{1}}^{\infty} \text { and } \phi_{2}=\phi_{2}^{\prime} \circ \pi_{n_{2}}^{\infty} \text {. }
$$

By Claim 1 and Lemma 2.1, $\phi_{1}^{\prime}$ and $\phi_{2}^{\prime}$ are epimorphisms. Now apply point (b) to the pair $D, D_{n_{1}}$, and the epimorphisms $\phi_{1}^{\prime}: D_{n_{1}} \rightarrow D$ and $\phi_{2}^{\prime}: D_{n_{2}} \rightarrow D$ to get $m>n_{2}$ (we can assume $m>n_{1}$ ) and an epimorphism $\chi: D_{m} \rightarrow D_{n_{1}}$ such that

$$
\phi_{1}^{\prime} \circ \chi=\phi_{2}^{\prime} \circ \pi_{n_{2}}^{m} \text {. }
$$

Applying Claim 2 we obtain an isomorphism $\psi$ of $\mathbb{D}$ with

$$
\chi \circ \pi_{m}^{\infty}=\pi_{n_{1}}^{\infty} \circ \psi \text {. }
$$

Now we have $\phi_{1}^{\prime} \circ \chi \circ \pi_{m}^{\infty}=\phi_{2}^{\prime} \circ \pi_{n_{2}}^{\infty}$ from (2.5) which yields $\phi_{1}^{\prime} \circ \pi_{n_{1}}^{\infty} \circ \psi=\phi_{2}^{\prime} \circ \pi_{n_{2}}^{\infty}$ by (2.6). From this it follows by (2.4) that $\psi$ is as required by (L3).

Now we prove (ii). Let $\mathbb{D}_{0}$ and $\mathbb{D}_{1}$ be two projective Fraïssé limits of $\Delta$. For $i=$ 0,1 , fix a sequence of clopen sets $\left(U_{n}^{i}\right)_{n \in \mathbb{N}}$ which separates points in $\mathbb{D}_{i}$ with $U_{0}^{0}=$ $U_{0}^{1}=\varnothing$. We construct a sequence $\left(D_{n}\right)_{n \in \mathbb{N}}$ of elements of $\Delta$ and epimorphisms $\phi_{n}^{i}: \mathbb{D}_{i} \rightarrow D_{n}$ and $\psi_{n}: D_{n+1} \rightarrow D_{n}$ such that

(c) $\phi_{n}^{i}=\psi_{n} \circ \phi_{n+1}^{i}$, for $i=0,1$ and $n \in \mathbb{N}$;

(d) $\phi_{2 n}^{0}$ refines the cover $\left\{U_{n}^{0}, \mathbb{D}_{0} \backslash U_{n}^{0}\right\}$ for $n \in \mathbb{N}$;

(e) $\phi_{2 n+1}^{1}$ refines the cover $\left\{U_{n}^{1}, \mathbb{D}_{1} \backslash U_{n}^{1}\right\}$ for $n \in \mathbb{N}$.

Pick $D_{0}$ to be any element of $\Delta$ and, by (L1) for $\mathbb{D}_{0}$, let $\phi_{0}^{0}$ be an epimorphism from $\mathbb{D}_{0}$ to $D_{0}$. Note that (d) holds since $U_{0}^{0}=\varnothing$. We will now show how to proceed from $2 n$ to $2 n+1$. (The step from $2 n+1$ to $2 n+2$ is identical.) Assume we have $D_{j}$ and $\phi_{j}^{0}$ for $j \leq 2 n$ and $\psi_{j}$ and $\phi_{j}^{1}$ for $j<2 n$. By Lemma 2.3 for $\mathbb{D}_{1}$ there is an epimorphism $\phi_{2 n}^{1}: \mathbb{D}_{1} \rightarrow D_{2 n}$ such that

$$
\psi_{2 n-1} \circ \phi_{2 n}^{1}=\phi_{2 n-1}^{1} \text {. }
$$


By Lemma 2.2, there is a $D_{2 n+1} \in \Delta$ and an epimorphism $\phi_{2 n+1}^{1}: \mathbb{D}_{1} \rightarrow D_{2 n+1}$ refining the open cover

$$
\left\{\left(\phi_{2 n}^{1}\right)^{-1}(d) \cap U_{n}^{1},\left(\phi_{2 n}^{1}\right)^{-1}(d) \cap\left(\mathbb{D}_{1} \backslash U_{n}^{1}\right): d \in D_{2 n}\right\} .
$$

The definition of the covering implies that there is a function $\psi_{2 n}: D_{2 n+1} \rightarrow D_{2 n}$ such that $\psi_{2 n} \circ \phi_{2 n+1}^{1}=\phi_{2 n}^{1}$. By Lemma $2.1 \psi_{2 n}$ is an epimorphism. Thus, (c) holds for $2 n$ and (e) for $n$. This finishes the inductive step.

We define a function $\phi: \mathbb{D}_{0} \rightarrow \mathbb{D}_{1}$ as follows. Take an $x \in \mathbb{D}_{0}$. By (c) and the surjectivity of each $\phi_{n}^{1}$, there is a $y \in \mathbb{D}_{1}$ such that for all $n, \phi_{n}^{0}(x)=\phi_{n}^{1}(y)$. By (e) this $y$ is unique. Let $\phi(x)=y$. By (c) and the surjectivity of each $\phi_{n}^{0}, \phi$ is onto. By $(\mathrm{d}), \phi$ is an injection. The very definition of $\phi$ makes it continuous.

Now we need to check that for $x_{1}, \ldots, x_{k}, y$ from $\mathbb{D}_{0}$

$$
\text { if }\left(x_{1}, \ldots, x_{k}\right) \in R^{\mathbb{D}_{0}} \text {, then }\left(\phi\left(x_{1}\right), \ldots, \phi\left(x_{k}\right)\right) \in R^{\mathbb{D}_{1}}
$$

and

$$
\text { if } f^{\mathbb{D}_{0}}\left(x_{1}, \ldots, x_{k}\right)=y \text {, then } f^{\mathbb{D}_{1}}\left(\phi\left(x_{1}\right), \ldots, \phi\left(x_{k}\right)\right)=\phi(y)
$$

for any $k$-ary relation symbol $R$ and any $k$-ary function symbol $f$. This suffices to see that $\phi$ is an isomorphism since, by symmetry, these same two conditions are then fulfilled by $\phi^{-1}$. Let us prove only the first condition for $\phi$. Assume towards contradiction that for some $x_{1}, \ldots, x_{k} \in \mathbb{D}_{0}$

$$
\left(x_{1}, \ldots, x_{k}\right) \in R^{\mathbb{D}_{0}} \text { and }\left(\phi\left(x_{1}\right), \ldots, \phi\left(x_{k}\right)\right) \notin R^{\mathbb{D}_{1}} .
$$

Since $R^{\mathbb{D}_{1}}$ is closed, by conditions (c) and (e) we can find $n$ and $d_{1}, \ldots, d_{k} \in D_{n}$ such that

$$
\left(\phi\left(x_{1}\right), \ldots, \phi\left(x_{k}\right)\right) \in\left(\phi_{n}^{1}\right)^{-1}\left(d_{1}\right) \times \cdots \times\left(\phi_{n}^{1}\right)^{-1}\left(d_{k}\right) \subseteq\left(\mathbb{D}_{1}\right)^{k} \backslash R^{\mathbb{D}_{1}} .
$$

Then by the definition of epimorphism, $\left(\phi_{n}^{1}\left(\phi\left(x_{1}\right)\right), \ldots, \phi_{n}^{1}\left(\phi\left(x_{k}\right)\right)\right) \notin R^{D_{n}}$. On the other hand, from (2.7) and the definition of epimorphism, $\left(\phi_{n}^{0}\left(x_{1}\right), \ldots, \phi_{n}^{0}\left(x_{k}\right)\right) \in$ $R^{D_{n}}$ which immediately leads to a contradiction since $\phi_{n}^{1}\left(\phi\left(x_{j}\right)\right)=\phi_{n}^{0}\left(x_{j}\right)$ for all $j \leq k$.

2.3. Additional properties. The content of the following lemma is that if $D$ is a topological $L$-structure each of whose open covers is refined by an epimorphism onto a structure from a family of finite topological $L$-structures $\Delta$, then $D$ can be regarded as an inverse limit of structures from $\Delta$.

Lemma 2.5. Let $\Delta$ be a family of finite topological L-structures. Let $D$ be a topological $L$-structure such that each open cover of $D$ is refined by an epimorphism from $D$ onto a structure in $\Delta$. Then there is a sequence $\left(D_{n}\right) \subseteq \Delta$ and epimorphisms $\psi_{n}: D_{n+1} \rightarrow D_{n}$ and $\phi_{n}: D \rightarrow D_{n}$ such that

(i) $\psi_{n} \circ \phi_{n+1}=\phi_{n}$;

(ii) each open cover of $D$ is refined by $\phi_{n}$ for some $n \in \mathbb{N}$.

Proof. Let $\mathcal{U}_{n}, n \in \mathbb{N}$, enumerate all clopen covers of $D$. Let $D_{0} \in \Delta$ and let $\phi_{0}: D \rightarrow D_{0}$ be an epimorphism refining $\mathcal{U}_{0}$. If $D_{n} \in \Delta$ and $\phi_{n}: D \rightarrow D_{n}$ has been defined, consider the cover

$$
\left\{U \cap \phi_{n}^{-1}(d): d \in D_{n} \text { and } U \in \mathcal{U}_{n}\right\} .
$$

Let $D_{n+1} \in \Delta$ and let $\phi_{n+1}: D \rightarrow D_{n+1}$ be an epimorphism refining the above cover. By the definition of the cover, there exists a function $\psi_{n}: D_{n+1} \rightarrow D_{n}$ such that $\psi_{n} \circ \phi_{n+1}=\phi_{n}$. By Lemma 2.1, $\psi_{n}$ is an epimorphism. 
Proposition 2.6. Let $\Delta$ be a projective Fraïssé family of finite topological Lstructures and let $\mathbb{D}$ be the projective Fraïssé limit of $\Delta$. Let $D$ be a topological $L$-structure such that any open covering of $D$ is refined by an epimorphism onto a structure in $\Delta$. Then there is an epimorphism from $\mathbb{D}$ onto $D$.

Proof. For $D$ fix $D_{n}, \phi_{n}$, and $\psi_{n}$ as in Lemma 2.5. By (L1) we can find an epimorphism $\phi_{0}^{\prime}: \mathbb{D} \rightarrow D_{0}$, and using Lemma 2.3 we can inductively find $\phi_{n+1}^{\prime}: \mathbb{D} \rightarrow D_{n+1}$ such that

$$
\psi_{n} \circ \phi_{n+1}^{\prime}=\phi_{n}^{\prime}
$$

We define a function $\phi: \mathbb{D} \rightarrow D$ as follows. Take an $x \in \mathbb{D}$. By (2.8) and since each $\phi_{n}^{\prime}$ is onto, there is a $y \in D$ such that for all $n, \phi_{n}^{\prime}(x)=\phi_{n}(y)$. By condition (ii) of Lemma 2.5 for the sequence $\left(\phi_{n}\right)$, this $y$ is unique. Let $\phi(x)=y$. By (2.8) and since each $\phi_{n}^{\prime}$ is onto, $\phi$ is onto. The definition of $\phi$ makes it continuous.

An argument as in the proof of Theorem 2.4(ii) justifies that for $x_{1}, \ldots, x_{k}, y \in \mathbb{D}$

$$
\text { if }\left(x_{1}, \ldots, x_{k}\right) \in R^{\mathbb{D}} \text {, then }\left(\phi\left(x_{1}\right), \ldots, \phi\left(x_{k}\right)\right) \in R^{D}
$$

and

$$
\text { if } f^{\mathbb{D}}\left(x_{1}, \ldots, x_{k}\right)=y \text {, then } f^{D}\left(\phi\left(x_{1}\right), \ldots, \phi\left(x_{k}\right)\right)=\phi(y)
$$

for any $k$-ary relation symbol $R$ and any $k$-ary function symbol $f$. We only need to verify that if $\left(y_{1}, \ldots, y_{k}\right) \in R^{D}$, then for some $x_{1}, \ldots, x_{k} \in \mathbb{D}$ with $\phi\left(x_{i}\right)=y_{i}$, for $i \leq k$, we have $\left(x_{1}, \ldots, x_{k}\right) \in R^{\mathbb{D}}$. Note that for each $n$ there are $x_{1}^{n}, \ldots, x_{k}^{n} \in \mathbb{D}$ such that $\phi_{n}^{\prime}\left(x_{i}^{n}\right)=\phi_{n}\left(y_{i}\right)$, for $i \leq k$, and $\left(x_{1}^{n}, \ldots, x_{k}^{n}\right) \in R^{\mathbb{D}}$. This is because $\phi_{n}$ and $\phi_{n}^{\prime}$ are epimorphisms. By compactness of $\mathbb{D}$, we can assume that the sequence $\left(x_{1}^{n}, \ldots, x_{k}^{n}\right)_{n}$ converges to some $\left(x_{1}, \ldots, x_{k}\right) \in \mathbb{D}^{k}$. By definition of $\phi$, we have $\phi\left(x_{i}\right)=y_{i}$, for $i \leq k$. Since $R^{\mathbb{D}}$ is closed, we get $\left(x_{1}, \ldots, x_{k}\right) \in R^{\mathbb{D}}$, as well.

2.4. Remarks. The classical Fraïssé construction is a method of taking a direct limit of a family of finite models of a language, provided the family fulfills certain conditions. The limit is a (countable) model of the same language which can be characterized by its (injective) homogeneity and universality with respect to the initial family of models. The standard example here is the family of finite linear orders. In this case the Fraïssé limit is the set of all rational numbers with the usual ordering. See [5] for a treatment.

The results in this section can be viewed as dual to this classical theory. Two points about the dualization need to be emphasized. First, our use of topology in the projective Fraïssé limit has no parallel in the traditional injective Fraïssé theory. Of course, the structures in the injective Fraïssé theory could just as well be equipped with the discrete topology, however, the topology would play no evident role. The situation is different when considering projective limits. If the projective limit is infinite, then it is non-discrete compact, and the topology plays an important role in the uniqueness of the projective Fraïssé limit and is crucial in applications. Second, readers familiar with the classical Fraïssé limit will notice a marked similarity between two of the conditions from that theory and the conditions in our definition of projective Fraïssé family. There is, however, in the classical theory a third condition which is absent from our definition, namely the Hereditary Property. In our case it is essential that we omit (the projective version of) this property since the families in the applications we have in mind do not fulfill it. 


\section{THE FAMILY OF FINITE LINEAR GRAPHS}

Let $L_{0}$ be the language consisting of one binary relation $R$. Given a topological $L_{0}$-structure $A$, we will henceforth write $R^{A}(a, b)$ to mean $(a, b) \in R^{A}$. Let $\Delta_{0}$ be the class of all finite (reflexive) linear graphs, i.e., the class of all finite sets $A$ with at least two elements so that $R^{A}$ has the following properties:

(1) $R^{A}$ is reflexive;

(2) $R^{A}$ is symmetric;

(3) every element of $A$ has at most three (including itself) $R^{A}$-neighbors;

(4) there are exactly two elements of $A$ with less than three $R^{A}$-neighbors;

(5) $R^{A}$ is connected, i.e., for every $a, b \in A$ there exists $a_{0}, \ldots, a_{n} \in A$ such that $a=a_{0}, b=a_{n}$, and $R^{A}\left(a_{i}, a_{i+1}\right)$ for $0 \leq i<n$.

Given $A \in \Delta_{0}$, a labeling of $A$ is a one-to-one function $l: A \rightarrow \mathbb{N}$ such that for each $a, b \in A$ with $a \neq b$ we have $R^{A}(a, b) \Leftrightarrow|l(a)-l(b)|=1$ and one endpoint, i.e., an element satisfying (4), of $A$ gets mapped by $l$ to 0 . Note that there are precisely two labelings on $A$. Once a labeling of $A$ has been given, for convenience we will regularly identify the points of $A$ with their labels. If $I \subseteq A$, then $\max (I)$ and $\min (I)$ will respectively mean the element of $I$ (or its label) with the maximum and minimum label. Note that labelings are for convenience only; in particular, a labeling of $A$ is not a part of the structure.

If $R^{A}(a, b)$ we will say that $a$ and $b$ are $R$-neighbors. We call $I \subseteq A$ an interval if for any $a, b \in I$, there are $a_{0}, \ldots, a_{n} \in I$ such that $a_{0}=a, a_{n}=b$, and $R^{A}\left(a_{i}, a_{i+1}\right)$ for all $i<n$. An element $a$ of $I$ is called an endpoint of $I$ if there are at most two elements $b \in I$ with $R^{A}(a, b)$ (one of which is, of course, $a$ itself). We say that two intervals $I$ and $J$ are adjacent if they are disjoint, and there is an endpoint $a$ of $I$ and an endpoint $b$ of $J$ such that $R^{A}(a, b)$.

Theorem 3.1. $\Delta_{0}$ is a projective Fraïssé class.

Proof. We need to show that $\Delta_{0}$ satisfies (F1) and (F2). Property (F1) is quite simple, and we leave it to the reader to check. We will prove (F2).

We start with describing the construction of an unfolding. Let $A$ be in $\Delta_{0}$ and let $I$ be a proper subinterval of $A$. The pair $\left(\tilde{A}, f_{A}\right)$, where $\tilde{A} \in \Delta_{0}$ and $f_{A}: \tilde{A} \rightarrow A$ is an epimorphism, will be called the unfolding of $A$ by $I$ if $|\tilde{A}|=|A|+2(|I|-1)$, and for some labelings on $A$ and $\tilde{A}$ we have

$$
f_{A}(i)= \begin{cases}i, & i \leq \max (I), \\ 2 \max (I)-i, & \max (I)<i<2 \max (I)-\min (I), \\ i-(2|I|-2), & 2 \max (I)-\min (I) \leq i .\end{cases}
$$

Note that given a labeling of $A$ if $\left(\tilde{A}, f_{A}\right)$ is an unfolding of $A$, then there is a labeling on $\tilde{A}$ such that (3.1) holds. Note also that if $J \subseteq A$ is an interval disjoint from $I$, then $f_{A}^{-1}(J)$ is an interval. If $I_{1}, \ldots, I_{n}$ are pairwise disjoint proper subintervals of $A$, then $\left(\tilde{A}, f_{A}\right)$ is the unfolding of $A$ by $I_{1}, \ldots, I_{n}$ if there is a sequence $A_{i}, 0 \leq i \leq n$, of structures from $\Delta_{0}$ and epimorphisms $f_{i}: A_{i} \rightarrow A_{i-1}$ such that for each $i \in\{1, \ldots, n\},\left(A_{i}, f_{i}\right)$ is an unfolding of $A_{i-1}$ by the interval $f_{i-1}^{-1} \circ \cdots \circ f_{1}^{-1}\left(I_{i}\right), A_{0}=A, A_{n}=\tilde{A}$, and $f_{A}=f_{1} \circ \cdots \circ f_{n}$. Note that the unfolding $\left(\tilde{A}, f_{A}\right)$ does not depend on the order of the intervals $I_{1}, \ldots, I_{n}$. Also note that if a labeling is given on $A$, then there is a labeling on $\tilde{A}$ such that $f_{A}(0)=0$. We will call such labelings compatible. 
Claim 1. Let $A$ and $B$ be in $\Delta_{0}$. Let $\phi: B \rightarrow A$ be an epimorphism. Let $J \subseteq A$ be a proper subinterval and let $\left(\tilde{A}, f_{A}\right)$ be the unfolding of $A$ by $J$. Then there exists a $\tilde{B} \in \Delta_{0}$ with epimorphisms $\pi: \tilde{B} \rightarrow B$ and $\tilde{\phi}: \tilde{B} \rightarrow \tilde{A}$ such that

$$
f_{A} \circ \tilde{\phi}=\phi \circ \pi \text {. }
$$

Proof of Claim 1, If $|J|=1$, note that $f_{A}: \tilde{A} \rightarrow A$ is an isomorphism, so identify $\tilde{A}$ with $A$ and let $\tilde{B}=B$, let $\pi$ be the identity on $B$, and let $\tilde{\phi}=\phi$.

Now suppose $|J| \geq 2$. Let $I_{1}, \ldots, I_{n}$ be the maximal subintervals of $\phi^{-1}(J)$, so they are pairwise disjoint, not adjacent, and their union is $\phi^{-1}(J)$. Let $\left(\tilde{B}, f_{B}\right)$ be the unfolding of $B$ by $I_{1}, \ldots, I_{n}$. Let $\pi=f_{B}$.

Label $A$ and $\tilde{A}$, and $B$ and $\tilde{B}$ with compatible labelings. There are three maximal subintervals of $\tilde{A}$ contained in $f_{A}^{-1}(J)$ on which $f_{A}$ is one-to-one. Let $\tilde{J}_{\text {bot }}$ be the one that contains $\min \left(f_{A}^{-1}(J)\right)$, let $\tilde{J}_{\text {top }}$ be the one that contains $\max \left(f_{A}^{-1}(J)\right)$, and let $\tilde{J}_{\text {mid }}$ be the one that contains neither $\min \left(f_{A}^{-1}(J)\right) \operatorname{nor} \max \left(f_{A}^{-1}(J)\right)$. For each $I_{i}$ with $\left|I_{i}\right| \geq 2$, let $\tilde{I}_{i \text { bot }}, \tilde{I}_{i \text { mid }}$, and $\tilde{I}_{i \text { top }}$ be subintervals of $\tilde{B}$ defined in an analogous fashion using $f_{B}$.

We now define $\tilde{\phi}$. If $b \in \tilde{B} \backslash f_{B}^{-1}\left(\phi^{-1}(J)\right)$, then let $\tilde{\phi}(b)=a$, where $a \in \tilde{A}$ is unique such that $\phi \circ f_{B}(b)=f_{A}(a)$. That is, on the non-folded parts of $B, \tilde{\phi}$ and $\phi$ agree. For the folded parts there are three cases.

Case 1. No endpoint of $I_{i}$ is mapped by $\phi$ to $\max (J)$. If $b \in f_{B}^{-1}\left(I_{i}\right)$, then let

$$
\tilde{\phi}(b)=\left(f_{A} \uparrow \tilde{J}_{\text {bot }}\right)^{-1} \circ \phi \circ f_{B}(b) .
$$

Case 2. No endpoint of $I_{i}$ is mapped by $\phi$ to $\min (J)$. If $b \in f_{B}^{-1}\left(I_{i}\right)$, then let

$$
\tilde{\phi}(b)=\left(f_{A} \uparrow \tilde{J}_{\text {top }}\right)^{-1} \circ \phi \circ f_{B}(b) .
$$

Case 3. The endpoints of $I_{i}$ are mapped by $\phi$ onto the endpoints of $J$. Note that in this case $\left|I_{i}\right| \geq 2$. If $\phi\left(\min \left(I_{i}\right)\right)=\min (J)$, then let

$$
\tilde{\phi}(b)= \begin{cases}\left(f_{A} \uparrow \tilde{J}_{\mathrm{bot}}\right)^{-1} \circ \phi \circ f_{B}(b), & b \in \tilde{I}_{\text {ibot }}, \\ \left(f_{A} \uparrow \tilde{J}_{\text {mid }}\right)^{-1} \circ \phi \circ f_{B}(b), & b \in \tilde{I}_{i \mathrm{mid}}, \\ \left(f_{A} \uparrow \tilde{J}_{\mathrm{top}}\right)^{-1} \circ \phi \circ f_{B}(b), & b \in \tilde{I}_{i \mathrm{top}} .\end{cases}
$$

If $\phi\left(\min \left(I_{i}\right)\right)=\max (J)$, then let

$$
\tilde{\phi}(b)= \begin{cases}\left(f_{A} \uparrow \tilde{J}_{\mathrm{bot}}\right)^{-1} \circ \phi \circ f_{B}(b), & b \in \tilde{I}_{\text {itop }}, \\ \left(f_{A} \uparrow \tilde{J}_{\mathrm{mid}}\right)^{-1} \circ \phi \circ f_{B}(b), & b \in \tilde{I}_{\text {imid }}, \\ \left(f_{A} \uparrow \tilde{J}_{\mathrm{top}}\right)^{-1} \circ \phi \circ f_{B}(b), & b \in \tilde{I}_{i \mathrm{bot}} .\end{cases}
$$

We leave it to the reader to check that $\tilde{\phi}: \tilde{B} \rightarrow \tilde{A}$ is an epimorphism. Formula (3.2) is then obvious from the definition of $\tilde{\phi}$.

We will now prove (F2). We proceed by induction on the size of $D$. If $|D|=|C|$, then $\phi_{1}$ is an isomorphism: let $F=E$, let $\psi_{2}$ be the identity, and let $\psi_{1}=\phi_{1}^{-1} \circ \phi_{2}$. Assume now that $|D|>|C|$. It will suffice to show the following claim.

Claim 2. Let $D, C \in \Delta_{0}$ be such that $|D|>|C|$. Let an epimorphism $\phi: D \rightarrow C$ be given. There exists a $D^{\prime} \in \Delta_{0}$ with $\left|D^{\prime}\right|<|D|$ and an epimorphism $\pi^{\prime}: D \rightarrow D^{\prime}$ such that

(a) $\phi^{\prime} \circ \pi^{\prime}=\phi$ for some epimorphism $\phi^{\prime}: D^{\prime} \rightarrow C$; 
(b) for any $F^{\prime} \in \Delta_{0}$ and any epimorphism $\psi^{\prime}: F^{\prime} \rightarrow D^{\prime}$ there is an $F \in \Delta_{0}$ with epimorphisms $\psi: F \rightarrow D$ and $\pi: F \rightarrow F^{\prime}$ such that

$$
\psi^{\prime} \circ \pi=\pi^{\prime} \circ \psi \text {. }
$$

To see that this is enough, let $\phi_{1}: D \rightarrow C$ and $\phi_{2}: E \rightarrow C$ be epimorphisms. Applying Claim 2 to $\phi_{1}$, we obtain $D^{\prime}, \pi^{\prime}$ and also an epimorphism $\phi_{1}^{\prime}: D^{\prime} \rightarrow C$ as in (a), that is, we have $\phi_{1}^{\prime} \circ \pi^{\prime}=\phi_{1}$. Then by our inductive assumption, there is an $F^{\prime} \in \Delta_{0}$ with epimorphisms $\psi_{1}^{\prime}: F^{\prime} \rightarrow D^{\prime}$ and $\psi_{2}^{\prime}: F^{\prime} \rightarrow E$ so that

$$
\phi_{1}^{\prime} \circ \psi_{1}^{\prime}=\phi_{2} \circ \psi_{2}^{\prime} \text {. }
$$

Now, condition (b) allows us to find $F \in \Delta_{0}$, and epimorphisms $\psi_{1}: F \rightarrow D$ and $\pi: F \rightarrow F^{\prime}$ such that

$$
\psi_{1}^{\prime} \circ \pi=\pi^{\prime} \circ \psi_{1}
$$

Now from (a), (3.4), and (3.3) we get

$$
\phi_{1} \circ \psi_{1}=\phi_{1}^{\prime} \circ \pi^{\prime} \circ \psi_{1}=\phi_{1}^{\prime} \circ \psi_{1}^{\prime} \circ \pi=\phi_{2} \circ\left(\psi_{2}^{\prime} \circ \pi\right) \text {. }
$$

If we let $\psi_{2}=\psi_{2}^{\prime} \circ \pi$, then $\phi_{1} \circ \psi_{1}=\phi_{2} \circ \psi_{2}$ as required.

Proof of Claim 2. For $A, B \in \Delta_{0}$, let us call an epimorphism $\alpha: B \rightarrow A$ simple if for each $x, y \in B$ we have that if $R^{B}(x, y)$ and $\alpha(x)=\alpha(y)$, then $x=y$.

We first consider the case where $\phi$ is simple.

We claim that there is a $D^{\prime} \in \Delta_{0}$ and a proper subinterval $J \subseteq D^{\prime}$ such that

$$
\left|D^{\prime}\right|<|D|
$$

and if $\left(\tilde{D}^{\prime}, f_{D^{\prime}}\right)$ is the unfolding of $D^{\prime}$ by $J$, then there are epimorphisms $\alpha_{1}: \tilde{D}^{\prime} \rightarrow$ $D$ and $\alpha_{2}: D \rightarrow D^{\prime}$ such that

$$
f_{D^{\prime}}=\alpha_{2} \circ \alpha_{1}
$$

and there is a $\phi^{\prime}: D^{\prime} \rightarrow C$ with

$$
\phi^{\prime} \circ \alpha_{2}=\phi .
$$

This will suffice to prove the claim for when $\phi$ is simple. To see it, let $\pi^{\prime}=\alpha_{2}$. Given $F^{\prime} \in \Delta_{0}$ and an epimorphism $\psi^{\prime}: F^{\prime} \rightarrow D^{\prime}$, by Claim 1 there is an $F \in \Delta_{0}$ with epimorphisms $\pi: F \rightarrow F^{\prime}$ and $\tilde{\psi}: F \rightarrow \tilde{D}^{\prime}$ such that $\psi^{\prime} \circ \pi=f_{D^{\prime}} \circ \tilde{\psi}$, that is, by (3.5), $\psi^{\prime} \circ \pi=\alpha_{2} \circ\left(\alpha_{1} \circ \tilde{\psi}\right)$. Thus, we can let $\psi=\alpha_{1} \circ \tilde{\psi}$.

Therefore, it remains to prove the existence of $D^{\prime}$ and $J$ as above. For the remainder of the proof fix some labelings on $D$ and $C$. Consider all maximal subintervals $J \subseteq D$ such that $\phi \uparrow J$ is one-to-one. Note that, since $\phi$ is simple, each such subinterval has at least two elements. Let $J_{1}$ be (one of) the shortest among such subintervals. Since $|D|>|C|, J_{1} \neq D$, and therefore we have one of the following cases.

Case 1. Neither endpoint of $J_{1}$ is an endpoint of $D$.

By the assumptions on $J_{1}$ and $\phi$, there are two disjoint subintervals $J_{0}$ and $J_{2}$ which are adjacent to $J_{1}$ and such that $\left|J_{0}\right|=\left|J_{2}\right|=\left|J_{1}\right|-1$, and $\phi \uparrow J_{0}$ and $\phi \uparrow J_{2}$ are one-to-one. Let $D^{\prime} \in \Delta_{0}$ be such that $\left|D^{\prime}\right|=|D|-2\left(\left|J_{1}\right|-1\right)$ and, for some labeling on $D^{\prime}$, let $f_{D^{\prime}}: D \rightarrow D^{\prime}$ be given by

$$
f_{D^{\prime}}(i)= \begin{cases}i, & 0 \leq i \leq \min \left(J_{1}\right), \\ 2 \min \left(J_{1}\right)-i, & \min \left(J_{1}\right)<i<\max \left(J_{1}\right), \\ i-2\left(\left|J_{1}\right|-1\right), & \max \left(J_{1}\right)<i \leq \max (D) .\end{cases}
$$


Put $J=f_{D^{\prime}}\left(J_{1}\right)$. Thus, we specified $D^{\prime}$ and $J$. Since $\left|J_{1}\right| \geq 2,\left|D^{\prime}\right|<|D|$. Note also that $J$ is a proper subinterval of $D^{\prime}$ and that $\left(D, f_{D^{\prime}}\right)$ is the unfolding of $D^{\prime}$ by $J$. Therefore, we can let $\alpha_{1}$ be the identity. It is easy to see that $\phi^{\prime}: D^{\prime} \rightarrow C$ given by the formula

$$
\phi^{\prime}(x)=\phi(y) \text { for any } y \in D \text { with } x=f_{D^{\prime}}(y)
$$

is well defined and is an epimorphism. Once this is established, it is clear that $\phi=\phi^{\prime} \circ f_{D^{\prime}}$. Thus, by letting $\alpha_{2}=f_{D^{\prime}}$, we get (3.5) and (3.6).

Case 2. Precisely one endpoint of $J_{1}$ is an endpoint of $D$.

Suppose without loss of generality that $\min \left(J_{1}\right)$ is an endpoint of $D$. By the case assumption there exists a subinterval $J_{2}$ of $D$ which is adjacent to $J_{1}$ such that $\left|J_{2}\right|=\left|J_{1}\right|-1$ and $\phi\left\lceil J_{2}\right.$ is one-to-one.

Let $D^{\prime} \in \Delta_{0}$ be such that $\left|D^{\prime}\right|=|D|-\left|J_{1}\right|+1$. Note that since $\left|J_{1}\right| \geq 2$, $\left|D^{\prime}\right|<|D|$. Define an epimorphism $\alpha_{2}: D \rightarrow D^{\prime}$ so that for some fixed labeling on $D^{\prime}$

$$
\alpha_{2}(i)= \begin{cases}\max \left(J_{1}\right)-i, & 0 \leq i \leq \max \left(J_{1}\right), \\ i-\max \left(J_{1}\right), & \max \left(J_{1}\right)<i \leq \max (D),\end{cases}
$$

Put $J=\alpha_{2}\left(J_{1}\right)$. Note that $J$ is a proper subinterval of $D$. Now let $\tilde{D}^{\prime} \in \Delta_{0}$ be such that $\left|\tilde{D}^{\prime}\right|=|D|+\left|J_{1}\right|-1$. Define an epimorphism $\alpha_{1}: \tilde{D}^{\prime} \rightarrow D$ so that for some fixed labeling on $\tilde{D}^{\prime}$

$$
\alpha_{1}(i)= \begin{cases}\max \left(J_{1}\right)-i, & 0 \leq i \leq \max \left(J_{1}\right), \\ i-\max \left(J_{1}\right), & \max \left(J_{1}\right)<i \leq \max \left(\tilde{D}^{\prime}\right) .\end{cases}
$$

Let $f_{D^{\prime}}=\alpha_{2} \circ \alpha_{1}$. Note that with this definition $\left(\tilde{D}^{\prime}, f_{D^{\prime}}\right)$ is the unfolding of $D^{\prime}$ by $J$. Thus, (3.5) holds. Now define $\phi^{\prime}: D^{\prime} \rightarrow C$ via

$$
\phi^{\prime}(x)=\phi(y) \text { for any } y \in D \text { with } x=\alpha_{2}(y) .
$$

We leave it to the reader to check the simple fact that $\phi^{\prime}$ is a well-defined epimorphism. Clearly (3.6) is fulfilled.

We proved the claim for simple $\phi: D \rightarrow C$. Assume now that $\phi$ is not simple and fix $d_{1}, d_{2} \in D$ such that $R^{D}\left(d_{1}, d_{2}\right), d_{1} \neq d_{2}$ and $\phi\left(d_{1}\right)=\phi\left(d_{2}\right)$. Let $D^{\prime} \in \Delta_{0}$ be such that $\left|D^{\prime}\right|=|D|-1$ and let $\pi: D \rightarrow D^{\prime}$ be an epimorphism with $\pi\left(d_{1}\right)=\pi\left(d_{2}\right)$. So $\pi \uparrow\left(D \backslash\left\{d_{i}\right\}\right), i=1,2$, is injective. Define $\phi^{\prime}(x)=\phi(y)$ for any $y \in D$ with $x=\pi(y)$. Then $\phi^{\prime}: D^{\prime} \rightarrow C$ is a well-defined epimorphism. Clearly (a) holds. Checking (b) is straightforward, and we leave it to the reader.

This proves the claim and, therefore, also the theorem.

\section{THE PSEUdO-ARC}

4.1. The projective Fraïssé limit of $\Delta_{0}$ and the pseudo-arc. Let $L_{0}$ be the language consisting of one binary relation $R$. Let $\Delta_{0}$ be the class of finite $L_{0^{-}}$ structures which are linear graphs as defined in the previous section.

Lemma 4.1. Let $\mathbb{P}$ be the projective Fraïssé limit of $\Delta_{0}$. Then $R^{\mathbb{P}}$ is an equivalence relation each of whose equivalence classes has at most two elements.

Proof. It suffices to show that $R^{\mathbb{P}}$ is reflexive, symmetric, and that for any $x \in \mathbb{P}$ there is at most one $y \in \mathbb{P}$ distinct from $x$ such that $R^{\mathbb{P}}(x, y)$. 
Let $x \in \mathbb{P}$. Since $R^{\mathbb{P}}$ is closed, to show that $R^{\mathbb{P}}(x, x)$, it will suffice to find in any clopen neighborhood $U$ of $x$ points $y_{1}$ and $y_{2}$ with $R^{\mathbb{P}}\left(y_{1}, y_{2}\right)$. Fix a clopen set $U$ containing $x$. Use Lemma 2.2 to get $D \in \Delta_{0}$ and an epimorphism $\phi: \mathbb{P} \rightarrow D$ refining $\{U, \mathbb{P} \backslash U\}$. Since $R^{D}(\phi(x), \phi(x))$, there are $y_{1}, y_{2}$ such that $\phi\left(y_{1}\right)=\phi(x)=\phi\left(y_{2}\right)$ and $R^{\mathbb{P}}\left(y_{1}, y_{2}\right)$. Clearly $y_{1}, y_{2} \in U$.

Let $x, y \in \mathbb{P}$ be such that $R^{\mathbb{P}}(x, y)$. Again to see that $R^{\mathbb{P}}(y, x)$, it suffices to prove the following. For any clopen sets $V, U$ containing $y$ and $x$, respectively, there is a $y_{1} \in V$ and an $x_{1} \in U$ so that $R^{\mathbb{P}}\left(y_{1}, x_{1}\right)$. Given such $V$ and $U$, let $D \in \Delta_{0}$ and $\phi: \mathbb{P} \rightarrow D$ be such that $\phi$ is an epimorphism refining $\{U, V, \mathbb{P} \backslash(U \cup V)\}$. Then $R^{D}(\phi(x), \phi(y))$, hence $R^{D}(\phi(y), \phi(x))$. It follows that there is a $y_{1}$ and an $x_{1}$ such that $R^{\mathbb{P}}\left(y_{1}, x_{1}\right), \phi\left(y_{1}\right)=\phi(y)$, and $\phi\left(x_{1}\right)=\phi(x)$. These $x_{1}$ and $y_{1}$ are as required.

Assume now towards contradiction that there are distinct $x, y, z \in \mathbb{P}$ such that $R^{\mathbb{P}}(x, y)$ and $R^{\mathbb{P}}(y, z)$. Let $U, V, W$ be clopen disjoint sets containing $x, y, z$, respectively. Let $D \in \Delta_{0}$ and $\phi: \mathbb{P} \rightarrow D$ be such that $\phi$ is an epimorphism refining $\{U, V, W, \mathbb{P} \backslash(U \cup V \cup W)\}$. Let $a, b, c \in D$ be the images via $\phi$ of $x, y, z$, respectively. Let $D^{\prime} \in \Delta_{0}$ and $\psi: D^{\prime} \rightarrow D$ be an epimorphism such that $\psi^{-1}(\{a, b, c\})=\left\{a^{\prime}, b^{\prime}, b^{\prime \prime}, c^{\prime}\right\}$ with $a^{\prime}, b^{\prime}, b^{\prime \prime}, c^{\prime}$ distinct and such that $\psi\left(a^{\prime}\right)=a$, $\psi\left(b^{\prime}\right)=\psi\left(b^{\prime \prime}\right)=b$, and $\psi\left(c^{\prime}\right)=c$ and $R^{D^{\prime}}\left(a^{\prime}, b^{\prime}\right), R^{D^{\prime}}\left(b^{\prime}, b^{\prime \prime}\right)$, and $R^{D^{\prime}}\left(b^{\prime \prime}, c^{\prime}\right)$. Note that then $\neg R^{D^{\prime}}\left(b^{\prime}, c^{\prime}\right)$ and $\neg R^{D^{\prime}}\left(a^{\prime}, b^{\prime \prime}\right)$. Now, by Lemma 2.3, there exists an epimorphism $\chi: \mathbb{P} \rightarrow D^{\prime}$ such that $\psi \circ \chi=\phi$. Note that we have $\chi(x)=a^{\prime}, \chi(z)=c^{\prime}$, and $\chi(y)$ is equal either to $b^{\prime}$ or to $b^{\prime \prime}$. But in the first case $\neg R^{D^{\prime}}(\chi(y), \chi(z))$ and in the second case $\neg R^{D^{\prime}}(\chi(x), \chi(y))$, leading to a contradiction.

The above lemma allows us to consider $\mathbb{P} / R^{\mathbb{P}}$ as a topological space with the quotient topology.

Theorem 4.2. Let $\mathbb{P}$ be the projective Fraïssé limit of $\Delta_{0}$. Then $\mathbb{P} / R^{\mathbb{P}}$ is a chainable hereditarily indecomposable continuum.

Thus by Bing's characterization of the pseudo-arc $\left[2, \mathbb{P} / R^{\mathbb{P}}\right.$ is the pseudo-arc.

We start with a lemma.

Lemma 4.3. Let $D$ be a topological $L_{0}$-structure such that $R^{D}$ is an equivalence relation. Assume that each open cover of $D$ is refined by an epimorphism from $D$ to an element of $\Delta_{0}$. Then $D / R^{D}$ is a chainable continuum.

Proof. Let $\rho: D \rightarrow D / R^{D}$ denote the quotient map. We first show that $D / R^{D}$ is compact, second countable, and connected, that is, that $D / R^{D}$ is a continuum. Compactness of $R^{D}$ easily implies that $D / R^{D}$ is Hausdorff. Then, continuity of $\rho$ gives that $D / R^{D}$ is compact with a countable basis since $D$ is such; see 4, Corollary 3.3.7]. That $D / R^{D}$ is connected will follow if we only show that given a non-empty clopen set $U \varsubsetneqq D$ there is an $x \in U$ and a $y \in D \backslash U$ such that $R^{D}(x, y)$. There is a $D_{0} \in \Delta_{0}$ and an epimorphism $\phi: D \rightarrow D_{0}$ refining the cover $\{U, D \backslash U\}$. Since $\phi(U) \cup \phi(D \backslash U)=D_{0}$, there is a $d_{1} \in \phi(U)$ and a $d_{2} \in \phi(D \backslash U)$ such that $R^{D_{0}}\left(d_{1}, d_{2}\right)$. This last condition ensures that there are $x, y \in D$ with $\phi(x)=d_{1}$, $\phi(y)=d_{2}$ and $R^{D}(x, y)$ since $\phi$ is an epimorphism. Since $\phi$ refines $\{U, D \backslash U\}$, we also have $x \in U$ and $y \in D \backslash U$.

Now we check chainability of $D / R^{D}$. Let $d$ be a compatible metric on $D / R^{D}$. Fix $\varepsilon>0$ and cover $D / R^{D}$ with a finite number of $\varepsilon$-balls $\left\{B_{0}, \ldots, B_{n}\right\}$. Now $\left\{\rho^{-1}\left(B_{0}\right), \ldots, \rho^{-1}\left(B_{n}\right)\right\}$ is an open cover of $D$. Let $\phi: D \rightarrow D_{0}$, for some $D_{0} \in \Delta_{0}$, be an epimorphism refining this cover. Let $U_{d}=\phi^{-1}(d)$ for $d \in D_{0}$. Note that 
each $U_{d}$ is clopen and that $\rho\left(U_{d_{1}}\right) \cap \rho\left(U_{d_{2}}\right) \neq \varnothing$ if and only if $R^{D_{0}}\left(d_{1}, d_{2}\right)$. Thus, by slightly enlarging each element of $\left\{\rho\left(U_{d}\right): d \in D_{0}\right\}$, we get a chain on $D / R^{D}$ refining $\left\{B_{0}, \ldots, B_{n}\right\}$, so the $d$-diameter of each of its elements is less than $\varepsilon$. Hence $D / R^{D}$ is chainable.

Proof of Theorem 4.2. The following notions will turn out to be useful in the proof. Let $D$ be a topological $L_{0}$-structure. A set $A \subseteq D$ is called $R$-invariant if for any $x \in A$ and $y \in D$, if $R^{D}(x, y)$ or $R^{D}(y, x)$, then $y \in A$. It is called $R$-connected if it is not a disjoint union of two non-empty, relatively closed sets $A_{1}$ and $A_{2}$ such that for all $x \in A_{1}$ and $y \in A_{2}$ neither $R^{D}(x, y)$ nor $R^{D}(y, x)$.

Note that by Lemma 2.2 each open covering of $\mathbb{P}$ is refined by an epimorphism onto an element of $\Delta_{0}$. Thus, by Lemma 4.3, $\mathbb{P} / R^{\mathbb{P}}$ is a chainable continuum. It remains to check that it is hereditarily indecomposable.

Let $\rho: \mathbb{P} \rightarrow \mathbb{P} / R^{\mathbb{P}}$ denote the quotient map. Let $X \subseteq \mathbb{P} / R^{\mathbb{P}}$ be a subcontinuum and suppose $X=X_{1} \cup X_{2}$ is the union of two proper subcontinua. Let $F=\rho^{-1}(X)$, $F_{1}=\rho^{-1}\left(X_{1}\right)$, and $F_{2}=\rho^{-1}\left(X_{2}\right)$. Note that $F_{1}$ and $F_{2}$ are $R$-connected and $R$ invariant. Furthermore, since $X_{1} \cap X_{2} \neq \varnothing, F_{1}$ and $F_{2}$ are not disjoint. It will suffice to show that either $F_{1} \subseteq F_{2}$ or $F_{2} \subseteq F_{1}$, since this will imply that either $X_{1} \subseteq X_{2}$ or $X_{2} \subseteq X_{1}$. Since $X, X_{1}, X_{2}$ are arbitrary, it will follow that $\mathbb{P} / R^{\mathbb{P}}$ is hereditarily indecomposable.

Suppose that $F_{1} \nsubseteq F_{2}$ and $F_{2} \nsubseteq F_{1}$; then there is an $x_{1} \in F_{1} \backslash F_{2}$ and an $x_{2} \in F_{2} \backslash F_{1}$. Since $F_{1}$ and $F_{2}$ are $R$-invariant, $\neg R^{\mathbb{P}}\left(x_{1}, y_{2}\right)$ for any $y_{2} \in F_{2}$ and $\neg R^{\mathbb{P}}\left(x_{2}, y_{1}\right)$ for any $y_{1} \in F_{1}$. Since $R^{\mathbb{P}}$ is closed and $F_{2}$ is compact, we can find clopen sets $U_{1}$ and $U_{2}$ such that $x_{1} \in U_{1}, F_{2} \subseteq U_{2}$ and $\neg R^{\mathbb{P}}\left(y_{1}, y_{2}\right)$ for any $y_{1} \in U_{1}$ and $y_{2} \in U_{2}$. Similarly we can find clopen sets $V_{1}$ and $V_{2}$ such that $F_{1} \subseteq V_{1}$, $x_{2} \in V_{2}$ and $\neg R^{\mathbb{P}}\left(y_{1}, y_{2}\right)$ for any $y_{1} \in V_{1}$ and $y_{2} \in V_{2}$. Let $\chi: \mathbb{P} \rightarrow D$, for some $D \in \Delta_{0}$, be an epimorphism refining the partition by the atoms of the algebra of sets generated by $U_{1}, U_{2}, V_{1}, V_{2}$.

Note now that since $F$ is $R$-connected, its image $\chi(F)$ is $R$-connected in $D$ and similarly for $F_{1}$ and $F_{2}$. Also note that since $F_{1}$ and $F_{2}$ are not disjoint, we have $\chi\left(F_{1}\right) \cap \chi\left(F_{2}\right) \neq \varnothing$. Obviously, we also have $\chi(F)=\chi\left(F_{1}\right) \cup \chi\left(F_{2}\right)$. Since $\chi^{-1}\left(\chi\left(x_{1}\right)\right) \subseteq U_{1}$ and $\chi^{-1}\left(\chi\left(F_{2}\right)\right) \subseteq U_{2}$ and since $\chi$ is an epimorphism, we see that $\chi\left(x_{1}\right) \notin \chi\left(F_{2}\right)$. (In fact, $\chi\left(x_{1}\right)$ is not even a neighbor of an element of $\chi\left(F_{2}\right)$.) Thus, $\chi\left(F_{1}\right) \backslash \chi\left(F_{2}\right) \neq \varnothing$. Similarly we obtain $\chi\left(F_{2}\right) \backslash \chi\left(F_{1}\right) \neq \varnothing$. By composing $\chi$ with an epimorphism from $D$ to an element of $\Delta_{0}$, we can assume that $\chi(F)=\left\{a_{0}, a_{1}, a_{2}\right\}$, where $R^{D}\left(a_{i}, a_{j}\right) \Leftrightarrow|i-j| \leq 1$, and that $\chi\left(F_{1}\right)=\left\{a_{0}, a_{1}\right\}$ and $\chi\left(F_{2}\right)=\left\{a_{1}, a_{2}\right\}$.

Let $B \in \Delta_{0}$ and $\phi: B \rightarrow D$ be chosen so that $\phi$ is an epimorphism, $\phi^{-1}(\chi(F))=$ $\left\{b_{0}, b_{1}, b_{2}, b_{3}, b_{4}, b_{5}, b_{6}\right\}$, where $R^{B}\left(b_{i}, b_{j}\right) \Leftrightarrow|i-j| \leq 1$, and

$$
\begin{aligned}
\phi\left(b_{0}\right) & =\phi\left(b_{4}\right)=a_{0}, \\
\phi\left(b_{1}\right)=\phi\left(b_{3}\right) & =\phi\left(b_{5}\right)=a_{1}, \\
\phi\left(b_{2}\right) & =\phi\left(b_{6}\right)=a_{2} .
\end{aligned}
$$

By Lemma 2.3, there is an epimorphism $\psi: \mathbb{P} \rightarrow B$ such that $\phi \circ \psi=\chi$. We now have that $\psi\left(F_{1}\right) \supseteq\left\{b_{0}, b_{4}\right\}$, but this contradicts the fact that $\psi\left(F_{1}\right)$ is $R$-connected and that $a_{2} \notin \chi\left(F_{1}\right)$.

4.2. Applications. In this subsection $P$ denotes the pseudo-arc and $\mathbb{P}$ denotes the projective Fraïssé limit of the family $\Delta_{0}$. 
This subsection contains applications of our results to the theory of the pseudoarc. First, in Theorem 4.4(i), we obtain from Theorem 4.2 and Proposition 2.6 a result of Mioduszewski [9] that each chainable continuum is a continuous image of the pseudo-arc. (Mioduszewski notes in [9] that his result seems to be derivable from an earlier theorem of Bing [1.) We then, in Theorem 4.4(ii), give a generalization of a result of Lewis [7] and Smith 13. who proved that homeomorphisms of the pseudo-arc are dense in the space of all continuous surjections from the pseudoarc to itself with the uniform convergence topology. (To see that this is a special case of (ii), note that given a continuous surjection $f: P \rightarrow P$, we can apply Theorem 4.4(ii) to $X=P, f_{1}=f, f_{2}=$ identity to get a homeomorphism $\varepsilon$ close to $f$.) These results are obtained as direct consequences of the general properties of projective Fraïssé limits. Moreover, following this line of thought it becomes clear that Theorems 4.2 and 2.4 suggest a new characterization of the pseudo-arc. We indeed establish such a characterization in Theorem 4.9 .

Theorem 4.4. (i) (Mioduszewski) Each chainable continuum is a continuous image of the pseudo-arc.

(ii) Let $X$ be a chainable continuum with a metric $d$ on it. If $f_{1}, f_{2}$ are continuous surjections from the pseudo-arc onto $X$, then for any $\varepsilon>0$ there exists a homeomorphism $h$ of the pseudo-arc such that $d\left(f_{1}(x), f_{2} \circ h(x)\right)<\varepsilon$ for all $x$.

Note that (L1) corresponds to (i) in the theorem above, (L2) corresponds to chainability of the pseudo-arc (see Lemma 4.3), and (L3) corresponds to (ii).

Lemma 4.3 suggests the following definition. A topological $L_{0}$-structure $D$ is called special if it fulfills the following conditions:

$(\alpha)$ each open cover of $D$ is refined by an epimorphism onto an element of $\Delta_{0}$;

( $\beta) R^{D}$ is an equivalence relation with each equivalence class having not more than two elements.

The following definition and lemma will allow us to transfer results about $\mathbb{P}$ to results about $P$. Let $D_{1}$ and $D_{2}$ be special topological $L_{0}$-structures. Let $\rho_{i}: D_{i} \rightarrow D_{i} / R^{D_{i}}, i=1,2$, denote the quotient functions. An epimorphism $\phi: D_{1} \rightarrow D_{2}$ induces a function $\phi^{*}: D_{1} / R^{D_{1}} \rightarrow D_{2} / R^{D_{2}}$ by $\phi^{*}\left(\rho_{1}(x)\right)=\rho_{2}(\phi(x))$. The fact that $\phi$ is an epimorphism implies that $\phi^{*}$ is well defined. We now have a lemma whose proof requires only checking definitions, so we leave the proof to the reader.

Lemma 4.5. Let $D_{1}$ and $D_{2}$ be special. Let $\phi: D_{1} \rightarrow D_{2}$ be an epimorphism. Let $\rho_{i}: D_{i} \rightarrow D_{i} / R^{D_{i}}, i=1,2$, denote the quotient functions. Then the following hold:

(i) $\phi^{*}$ is a continuous surjection from $D_{1} / R^{D_{1}}$ to $D_{2} / R^{D_{2}}$ and $\rho_{2} \circ \phi=\phi^{*} \circ \rho_{1}$;

(ii) if $\phi$ is an isomorphism, then $\phi^{*}$ is a homeomorphism.

We will now review some elementary facts about chainable compact metric spaces. For a metric space $(X, d)$ and non-empty sets $A, B \subseteq X$ we write

$$
\operatorname{dist}(A, B)=\inf \{d(a, b): a \in A, b \in B\}
$$

and

$$
\operatorname{diam}(A)=\sup \left\{d\left(a_{1}, a_{2}\right): a_{1}, a_{2} \in A\right\}
$$


Let $(X, d)$ be a chainable compact metric space. Let $\delta>0$. A chain $U_{1}, \ldots, U_{N}$ on $X$ is called a $\delta$-fine chain if

(C1) $\operatorname{dist}\left(U_{i}, U_{j}\right)>\delta$ if $|i-j|>1$;

(C2) $\operatorname{dist}\left(\{x\}, \bigcup_{j \neq i} U_{j}\right)>\delta$ for some $x \in U_{i}$;

(C3) for each $A \subseteq X$ with $\operatorname{diam}(A)<\delta$ there exists $i \leq N$ with $A \subseteq U_{i}$.

A chain is fine if it is $\delta$-fine for some $\delta>0$. A chain $U_{1}, \ldots, U_{N}$ closure refines a covering $\mathcal{V}$ if the closure of each $U_{i}$ is included in some element of $\mathcal{V}$. The following fact is easy to check.

Lemma 4.6. If $(X, d)$ is a chainable continuum, then each open cover of $X$ is closure refined by a fine chain.

The following lemma gives a converse to Lemma 4.3 .

Lemma 4.7. If $X$ is a chainable continuum, then there is a special topological $L_{0}$-structure $C$ such that $X$ is homeomorphic to $C / R^{C}$.

Proof. If $\mathcal{C}$ is a chain on $X$, we will denote the $k$-th element of $\mathcal{C}$, which we call its $k$-th link, by $\mathcal{C}(k)$. In particular, $\mathcal{C}(i) \cap \mathcal{C}(j) \neq \varnothing$ holds if and only if $|i-j| \leq 1$. Whenever we write $\mathcal{C}(k)$ we assume that $\mathcal{C}$ has at least $k$ links. If $\mathcal{C}$ is a chain, then the mesh of $\mathcal{C}$ is $\operatorname{mesh}(\mathcal{C})=\max \{\operatorname{diam}(\mathcal{C}(i)): 1 \leq i \leq N\}$, where $N$ is the number of links in $\mathcal{C}$.

We will construct a sequence of chains $\left(\mathcal{C}_{n}\right)_{n=0}^{\infty}$ on $X$ such that for each $n \in \mathbb{N}$

(1) $\mathcal{C}_{n+1}$ closure refines $\mathcal{C}_{n}$;

(2) $\operatorname{mesh}\left(\mathcal{C}_{n}\right)<\frac{1}{n}$;

(3) $\mathcal{C}_{n}$ is fine;

(4) if $\mathcal{C}_{n+1}(i) \subseteq \mathcal{C}_{n}(k), \mathcal{C}_{n+1}(j) \subseteq \mathcal{C}_{n}(l)$, and $|k-l|>1$, then $|i-j|>2$;

(5) for each $k$ there exists an $i$ such that $\mathcal{C}_{n+1}(i) \subseteq \mathcal{C}_{n}(k) \backslash \bigcup_{l \neq k} \mathcal{C}_{n}(l)$.

Let $\mathcal{C}_{0}$ be a fine chain on $X$. Now suppose we have $\mathcal{C}_{n}$ for $n \geq 0$; we will construct $\mathcal{C}_{n+1}$. Let $\delta>0$ be such that $\mathcal{C}_{n}$ is $\delta$-fine. Cover $X$ by balls of radius less than $\min (1 / 2(n+1), \delta / 6)$. Let $\mathcal{C}_{n+1}$ be a fine chain closure refining this cover. Observe that for each link $\mathcal{C}_{n+1}(i)$ of $\mathcal{C}_{n+1}$

$$
\operatorname{diam}\left(\overline{\mathcal{C}_{n+1}(i)}\right)<\delta / 3
$$

so, by (C3), $\mathcal{C}_{n+1}$ closure refines $\mathcal{C}_{n}$. Condition (2) is clear. We also observe that by (4.1) and (C1) we have (4). Condition (5) follows from (C2) and (4.1).

For each $n \in \mathbb{N}$ let $C_{n}=\{1, \ldots, N\}$, where $\mathcal{C}_{n}$ consists of $N$ links, and we take $R^{C_{n}}(i, j)$ precisely when $|i-j| \leq 1$, so in particular $R^{C_{n}}(i, j)$ iff $\mathcal{C}_{n}(i) \cap \mathcal{C}_{n}(j) \neq \varnothing$. For each $n \in \mathbb{N}$ define $\phi_{n}: C_{n+1} \rightarrow C_{n}$ via

$$
\phi_{n}(i)=\min \left\{k: \overline{\mathcal{C}_{n+1}(i)} \subseteq \mathcal{C}_{n}(k)\right\} .
$$

The function $\phi_{n}$ is well defined by (1). By (5), $\phi_{n}$ is onto, and it is an epimorphism by (4).

Let $C=\lim \left(C_{n}, \phi_{n}\right)$. We take each $C_{n}$ with the discrete topology and $C$ with the inverse limit topology. If $x \in C$, we will denote the natural projection of $x$ onto $C_{n}$ by $x(n)$, so in particular one can write $x=(x(0), x(1), x(2), \ldots)$. Define $R^{C}$ by letting $R^{C}(x, y)$ if and only if $R^{C_{n}}(x(n), y(n))$ for all $n$. 
Claim 1. $C$ is a special topological $L_{0}$-structure.

Proof of Claim 1. Clearly $C$ is a topological $L_{0}$-structure. We check that $R^{C}$ is an equivalence relation with each equivalence class having not more than two elements. It is clear that $R^{C}$ is reflexive and symmetric. Suppose $x, y, z \in C$ are such that $R^{C}(x, y)$ and $R^{C}(y, z)$ with $x \neq z$. First note that by $(4)$, for every $n \in \mathbb{N}, \phi_{n}^{-1}(y(n))$ contains $y(n+1)$ along with an $R$-neighbor of this element distinct from it. Since $R^{C_{n+1}}(x(n+1), y(n+1))$ and $R^{C_{n+1}}(y(n+1), z(n+1))$, unless $x(n+1)=z(n+1)$ we have $z(n+1) \in \phi_{n}^{-1}(y(n))$ or $x(n+1) \in \phi_{n}^{-1}(y(n))$. Therefore, we have $x(n)=y(n)$ or $z(n)=y(n)$ or $x(n)=z(n)$ for each $n$.

Now since $x \neq z$, for all but finitely many $n, x(n) \neq z(n)$. It must then be that for all but finitely many $n x(n)=y(n)$ or $z(n)=y(n)$. Thus, one of these possibilities is realized for all $n$, whence either $x=y$ or $z=y$. This proves the claim.

Claim 2. $X$ is homeomorphic to $C / R^{C}$.

Proof of Claim 2, Define $f: C \rightarrow X$ by letting $f(x)$ be the unique, by (1) and (2), element in $\bigcap_{n} \overline{\mathcal{C}_{n}(x(n))}$. It is a routine check that $f$ is continuous. To see that it is surjective fix $y \in X$. Consider the set $T_{y}$ of all sequences $\left(m_{0}, \ldots, m_{n}\right) \in \prod_{j \leq n} C_{j}$ such that $y \in \mathcal{C}_{n}\left(m_{n}\right)$ and $\phi_{j}\left(m_{j+1}\right)=m_{j}$ for $j<n$, where $n$ ranges over $\mathbb{N}$. Equip $T_{y}$ with the partial order of extension. Then $T_{y}$ is a tree. It is obviously finitely branching. As is easily seen, for each $n$ there exists an element of $T_{y}$ of length $n$; thus, $T_{y}$ is infinite. It follows now from König's lemma that there exists $x=\left(m_{0}, m_{1}, \ldots\right)$ such that for each $n$ we have $\left(m_{0}, \ldots, m_{n}\right) \in T_{y}$. Clearly then $x \in C$ and $f(x)=y$. Thus, $f$ is surjective.

We now check that

$$
f\left(x_{1}\right)=f\left(x_{2}\right) \Leftrightarrow R^{C}\left(x_{1}, x_{2}\right) .
$$

The implication $\Leftarrow$ follows immediately from $(2)$. To see $\Rightarrow$, note that $f\left(x_{1}\right)=f\left(x_{2}\right)$ is in $\bigcap_{n} \overline{\mathcal{C}_{n}\left(x_{i}(n)\right)}=\bigcap_{n} \mathcal{C}_{n}\left(x_{i}(n)\right)$ for $i=1,2$. Thus, for each $n, \mathcal{C}_{n}\left(x_{1}(n)\right)$ and $\mathcal{C}_{n}\left(x_{2}(n)\right)$ have a point in common. It follows that for each $n, R^{C_{n}}\left(x_{1}(n), x_{2}(n)\right)$, and we are done.

Now (4.2) allows us to define $\bar{f}: C / R^{C} \rightarrow X$ by letting $\bar{f}(\rho(x))=f(x)$ for $x \in C$. By [4, Proposition 2.4.2], $\bar{f}$ is a homeomorphism.

This completes the proof of the claim and hence the lemma.

Lemma 4.8. Identify $P$ with $\mathbb{P} / R^{\mathbb{P}}$. Let $X$ be a chainable continuum with a metric $d$ on it. Let $f_{1}$ and $f_{2}$ be continuous surjections from $P$ to $X$. Then, for any $\varepsilon>0$, there is an isomorphism $\phi: \mathbb{P} \rightarrow \mathbb{P}$ such that $d\left(f_{1}(x), f_{2}\left(\phi^{*}(x)\right)\right)<\varepsilon$ for any $x \in P$.

Proof. Let $\rho: \mathbb{P} \rightarrow P$ be the quotient map.

By Lemma 4.6] we can find a $\delta$-fine chain $U_{1}, \ldots, U_{N}$ for some $\delta>0$ which refines the covering of $X$ by balls of radius less than $\varepsilon / 2$. Thus, we have

$$
\operatorname{diam}\left(U_{i}\right)<\varepsilon \text { for } i \leq N \text {. }
$$

By Lemma 2.2 and the fact that $f_{1} \circ \rho$ and $f_{2} \circ \rho$ are uniformly continuous on $\mathbb{P}$, there are $E_{i} \in \Delta_{0}, i=1,2$, and epimorphisms $\phi_{i}: \mathbb{P} \rightarrow E_{i}$ such that

$$
\operatorname{diam}\left(f_{i} \circ \rho\left(\phi_{i}^{-1}(e)\right)\right)<\delta \text { for } e \in E_{i} .
$$


Let $D=\{1, \ldots, N\}$ with $R^{D}(i, j)$ precisely when $|i-j| \leq 1$. Then $D$ with $R^{D}$ is an element of $\Delta_{0}$. Now define $\psi_{i}: E_{i} \rightarrow D, i=1,2$, by letting

$$
\psi_{i}(e)=\min \left\{k \in\{1, \ldots, N\}: f_{i} \circ \rho\left(\phi_{i}^{-1}(e)\right) \subseteq U_{k}\right\} .
$$

By (4.4) and (C3), $\psi_{i}$ is well-defined. By (4.4), the fact that $f_{i}$ is a surjection, and (C2), $\psi_{i}$ is a surjection. To see that $\psi_{i}$ is an epimorphism, it suffices to show that if $e_{1}, e_{2} \in E_{i}$ and $R^{E_{i}}\left(e_{1}, e_{2}\right)$, then $R^{D}\left(\psi_{i}\left(e_{1}\right), \psi_{i}\left(e_{2}\right)\right)$, that is, $\left|\psi_{i}\left(e_{1}\right)-\psi_{i}\left(e_{2}\right)\right| \leq 1$. If $R^{E_{i}}\left(e_{1}, e_{2}\right)$, then, since $\phi_{i}$ is an epimorphism, there are $x_{1}, x_{2} \in \mathbb{P}$ with $\phi_{i}\left(x_{1}\right)=e_{1}$, $\phi_{i}\left(x_{2}\right)=e_{2}$, and $R^{\mathbb{P}}\left(x_{1}, x_{2}\right)$. This last condition gives $\rho\left(x_{1}\right)=\rho\left(x_{2}\right)$ from which it follows that

$$
f_{i} \circ \rho\left(\phi_{i}^{-1}\left(e_{1}\right)\right) \cap f_{i} \circ \rho\left(\phi_{i}^{-1}\left(e_{2}\right)\right) \neq \varnothing .
$$

Thus, by (4.4) and (C1), $\left|\psi_{i}\left(e_{1}\right)-\psi_{i}\left(e_{2}\right)\right| \leq 1$.

Now by (L3), there exists an isomorphism $\phi: \mathbb{P} \rightarrow \mathbb{P}$ such that for all $x \in \mathbb{P}$ we have

$$
\psi_{1} \circ \phi_{1}(x)=\psi_{2} \circ \phi_{2} \circ \phi(x) .
$$

This means that for each $x \in \mathbb{P}$ there exists $i \leq N$ such that $f_{1} \circ \rho(x), f_{2} \circ \rho(\phi(x)) \in$ $U_{i}$ which combined with (4.3) and Lemma 4.5(i) gives the conclusion of the lemma.

Proof of Theorem 4.4. (i) Let $X$ be a chainable continuum and, by Lemma 4.7 let $C$ be a special topological $L_{0}$-structure such that $C / R^{C}$ is homeomorphic to $X$. Then by Proposition 2.6 there is an epimorphism $\phi: \mathbb{P} \rightarrow C$. Now, by Theorem 4.2 and Lemma 4.5(i), $\phi^{*}$ is a continuous surjection from the pseudo-arc onto $X$.

(ii) is an immediate consequence of Lemma 4.8 and Lemma 4.5(ii).

As an analogue of Theorem 2.4 we have the following result. It gives an apparently new characterization of the pseudo-arc.

Theorem 4.9. The pseudo-arc is the unique non-degenerate chainable continuum $X$ such that for any chainable continuum $Y$ with a metric d, any continuous surjections $f_{1}, f_{2}$ from $X$ onto $Y$, and any $\varepsilon>0$ there exists a homeomorphism $h: X \rightarrow X$ such that $d\left(f_{1}(x), f_{2} \circ h(x)\right)<\varepsilon$ for all $x \in X$.

If $X$ and $Y$ are compact metric spaces with metrics $d_{X}$ and $d_{Y}$ respectively, then a continuous map $f: X \rightarrow Y$ is called a $\delta$-map if $\operatorname{diam}\left(f^{-1}(f(x))\right)<\delta$ for each $x \in X$. A basic fact about $\delta$-maps is the following: if $f: X \rightarrow Y$ is a $\delta$-map, then there is a $\zeta>0$ such that $\operatorname{diam}\left(f^{-1}(A)\right)<\delta$ whenever $\operatorname{diam}(A)<\zeta$ for any $A \subseteq Y$, so in particular if $d_{X}\left(x_{0}, x_{1}\right) \geq \delta$, then $d_{Y}\left(f\left(x_{0}\right), f\left(x_{1}\right)\right) \geq \zeta$. It is well known that a non-degenerate continuum $\left(X, d_{X}\right)$ is chainable if and only if for every $\delta>0$ there is a $\delta$-map from $X$ onto the closed unit interval; see [11, Theorem 12.11].

Below $|\cdot|$ stands for the absolute value on the reals.

Lemma 4.10 ([11, Lemma 12.17]). If $X$ is a compact metric space, $f: X \rightarrow[0,1]$ is a continuous surjection, and $\varepsilon>0$, then there exists a $\delta=\delta(f, \varepsilon)$ so that if $g: X \rightarrow[0,1]$ is any onto $\delta$-map, then there is a continuous surjection 1$]:[0,1] \rightarrow$ $[0,1]$ so that $|\phi \circ g(x)-f(x)|<\varepsilon$ for every $x \in X$.

\footnotetext{
${ }^{1}$ This lemma in [11 explicitly states that $\phi$ need not be onto, but an analysis of the proof shows that if $f$ is onto, then $\phi$ is onto.
} 
Proof of Theorem 4.9. The pseudo-arc fulfills the conclusion of the theorem by Theorem 4.4(ii).

Let $\left(X, d_{X}\right)$ and $\left(Y, d_{Y}\right)$ be chainable continua satisfying the condition from the statement of Theorem 4.9, For two functions $f$ and $g$ defined on the same set $Z$ with values in $[0,1]$, we write

$$
\|f-g\|=\sup _{z \in Z}|f(z)-g(z)| .
$$

Below $I_{n}$ is the unit interval for $n \in \mathbb{N}$. We will construct continuous surjections $\phi_{n}: I_{n+1} \rightarrow I_{n}$, continuous surjections $f_{n}: X \rightarrow I_{n}$ and $g_{n}: Y \rightarrow I_{n}$, and $\varepsilon_{n}>0$ such that for $n \geq 0$

$(a)_{n} \varepsilon_{n}<\frac{1}{n+1}$

(b) $n\left\|\phi_{k, n-1} \circ f_{n}-\phi_{k, m-1} \circ f_{m}\right\|<\varepsilon_{m}$ for all $k \leq m \leq n$;

$(c)_{n}\left\|\phi_{k, n-1} \circ g_{n}-\phi_{k, m-1} \circ g_{m}\right\|<\varepsilon_{m}$ for all $k \leq m \leq n$;

$(d)_{n}$ if $n$ is even and if $d_{X}(x, y) \geq \frac{1}{n+1}$, then $\left|f_{n}(x)-f_{n}(y)\right|>2 \varepsilon_{n}$, for $x, y \in X$;

$(e)_{n}$ if $n$ is odd and if $d_{Y}(x, y) \geq \frac{1}{n+1}$, then $\left|g_{n}(x)-g_{n}(y)\right|>2 \varepsilon_{n}$, for $x, y \in Y$, where we adopt the following notation: $\phi_{i, j}=\phi_{i} \circ \cdots \circ \phi_{j}: I_{j+1} \rightarrow I_{i}$, for $i \leq j$, with the convention that $\phi_{i, i-1}$ is the identity map on $I_{i}$.

Let $f_{0}: X \rightarrow I_{0}$ be a continuous surjection and let $\varepsilon_{0}$ be any positive real less than 1 such that for any $x, y \in X$ with $d_{X}(x, y) \geq 1,\left|f_{0}(x)-f_{0}(y)\right|>2 \varepsilon_{0}$. Clearly such an $\varepsilon_{0}$ can be found and, obviously, $(a)_{0}-(e)_{0}$ hold $\left((c)_{0}\right.$ and $(e)_{0}$ hold vacuously no matter how $g_{0}$ is chosen).

Assume now that we have chosen $\varepsilon_{i}$ and $f_{i}$ for $i \leq 2 n$ and $\phi_{i}$ and $g_{i}$ for $i<2 n$ so that they fulfill $(a)_{i},(b)_{i}$ and $(d)_{i}$ for $i \leq 2 n$ and $(c)_{i}$ and $(e)_{i}$ for $i<2 n$. We show now how to find $g_{2 n}, \phi_{2 n}, g_{2 n+1}$, and $\varepsilon_{2 n+1}$. (This is a half of the inductive step. The other half producing $f_{2 n+1}, \phi_{2 n+1}, f_{2 n+2}$ and $\varepsilon_{2 n+2}$ is essentially identical.) Since $Y$ satisfies the condition from the statement of Theorem 4.9. we have the following claim which is seen to hold by considering the surjections $f_{1}=g: Y \rightarrow[0,1]$ and $f_{2}=\phi \circ g: Y \rightarrow[0,1]$.

Claim. For any $\varepsilon>0$, given any continuous surjections $\phi:[0,1] \rightarrow[0,1]$ and $g$ : $Y \rightarrow[0,1]$, there is a continuous surjection $g^{\prime}: Y \rightarrow[0,1]$ such that $\left\|\phi \circ g^{\prime}-g\right\|<\varepsilon$.

Now note that by our inductive assumption $(c)_{2 n-1}$, we can find $\varepsilon>0$ such that for all $k \leq m \leq 2 n-1$ we have

$$
\left\|\phi_{k, 2 n-2} \circ g_{2 n-1}-\phi_{k, m-1} \circ g_{m}\right\|+\varepsilon<\varepsilon_{m} .
$$

By the claim, we can choose $g_{2 n}: Y \rightarrow I_{2 n}$ so that $\left\|\phi_{2 n-1} \circ g_{2 n}-g_{2 n-1}\right\|$ is as small as we wish, and we wish it to be small enough to fulfill

$$
\begin{aligned}
\| \phi_{k, 2 n-1} \circ g_{2 n} & -\phi_{k, 2 n-2} \circ g_{2 n-1} \| \\
& =\left\|\phi_{k, 2 n-2} \circ\left(\phi_{2 n-1} \circ g_{2 n}\right)-\phi_{k, 2 n-2} \circ g_{2 n-1}\right\|<\varepsilon
\end{aligned}
$$

for all $k \leq 2 n-1$. This is possible by uniform continuity of $\phi_{k, 2 n-2}$. But then (4.5), (4.6), and the triangle inequality give

$$
\left\|\phi_{k, 2 n-1} \circ g_{2 n}-\phi_{k, m-1} \circ g_{m}\right\|<\varepsilon_{m}
$$

for all $k \leq m \leq 2 n-1$. Since this inequality obviously also holds for $m=2 n$, we see that $g_{2 n}$ fulfills $(c)_{2 n}$. We now define $g_{2 n+1}, \phi_{2 n}$, and $\varepsilon_{2 n+1}$. Using the inductive assumption $(c)_{2 n}$, we can find $\varepsilon>0$ such that for all $k \leq m \leq 2 n$,

$$
\left\|\phi_{k, 2 n-1} \circ g_{2 n}-\phi_{k, m-1} \circ g_{m}\right\|+\varepsilon<\varepsilon_{m} \text {. }
$$


We will find $g_{2 n+1}$ and $\phi_{2 n}$ so that for all $k \leq 2 n$

$$
\begin{aligned}
\| \phi_{k, 2 n} \circ g_{2 n+1} & -\phi_{k, 2 n-1} \circ g_{2 n} \| \\
& =\left\|\phi_{k, 2 n-1} \circ\left(\phi_{2 n} \circ g_{2 n+1}\right)-\phi_{k, 2 n-1} \circ g_{2 n}\right\|<\varepsilon .
\end{aligned}
$$

Since $Y$ is chainable, we can pick $g_{2 n+1}: Y \rightarrow I_{2 n+1}$ to be a $\delta$-map with $\delta<$ $1 /(2 n+2)$ and small enough so that, by Lemma 4.10, we can choose a continuous surjection $\phi_{2 n}: I_{2 n+1} \rightarrow I_{2 n}$ making the quantity $\left\|\phi_{2 n} \circ g_{2 n+1}-g_{2 n}\right\|$ as small as we wish; in particular, small enough for (4.8) to hold. Now by the triangle inequality, using (4.7) and (4.8), we get that for all $k \leq m \leq 2 n$,

$$
\left\|\phi_{k, 2 n} \circ g_{2 n+1}-\phi_{k, m-1} \circ g_{m}\right\|<\varepsilon_{m} .
$$

Since as long as we choose $\varepsilon_{2 n+1}$ positive this inequality also holds for $m=2 n+1$, we see that $g_{2 n+1}$ and $\phi_{2 n}$ fulfill $(c)_{2 n+1}$. Since $g_{2 n+1}$ is a $\delta$-map for some $\delta<1 /(2 n+2)$, there exists $\varepsilon_{2 n+1}>0$ for which $(e)_{2 n+1}$ and $(a)_{2 n+1}$ hold. Thus, the construction is completed.

We will now show that $X$ and $Y$ are homeomorphic by showing that they are both homeomorphic to $Z=\lim \left(I_{n}, \phi_{n}\right)$. We prove this for $X$. Note that by $(a)_{n}$ and $(b)_{n}$ the sequence of functions $\left(\phi_{k, n-1} \circ f_{n}\right)_{n \geq k}$ converges uniformly to a continuous function $\bar{f}_{k}: X \rightarrow I_{k}$. Moreover, by $(b)_{n}$, for all $k$ and all $m \geq k$,

$$
\left\|\bar{f}_{k}-\phi_{k, m-1} \circ f_{m}\right\| \leq \varepsilon_{m} .
$$

From the identity $\phi_{k} \circ \phi_{k+1, n-1}=\phi_{k, n-1}$ for $n \geq k+1$ and from continuity of the functions $\phi_{k}$, we get that the image of the function $F: X \rightarrow \prod_{n} I_{n}$ given by $F(x)=\left(\bar{f}_{0}(x), \bar{f}_{1}(x), \ldots\right)$ is included in $Z$. Since each $\bar{f}_{k}$ is continuous, $F$ is continuous as well. To check that $F$ is onto it suffices to see that the range of $F$ is dense in $Z$, which will follow if we show that for each $k$ the range of $\bar{f}_{k}$ is dense in $I_{k}$. But this is immediate from surjectivity of $\phi_{k, m-1} f_{m}$, (4.9), and $(a)_{m}$. Thus, since $X$ is compact, it remains to show that $F$ is injective. Fix $x, y \in X$. Find $k_{0}$ with $d_{X}(x, y) \geq 1 /\left(2 k_{0}+1\right)$. From $(d)_{2 k_{0}}$ we have that $\left|f_{2 k_{0}}(x)-f_{2 k_{0}}(y)\right|>2 \varepsilon_{2 k_{0}}$. It follows now from (4.9) for $k=m=2 k_{0}$ that $\bar{f}_{2 k_{0}}(x) \neq \bar{f}_{2 k_{0}}(y)$, whence $F(x) \neq$ $F(y)$.

4.3. Remarks. 1. We would like to point out certain similarities between the theorems proved in this section and the theory developed by Bing and Moise. One can view this latter theory as follows. A chainable, hereditarily indecomposable continuum $P_{1}$ is constructed. Two results are proved about it: it is homogeneous [1], 10], i.e., for any two points $x, y \in P_{1}$ there is a homeomorphism of $P_{1}$ mapping $x$ to $y$ (and even more homogeneous by [6]), and it is approximately universal among chainable continua 2 , i.e., each chainable continuum can be approximated by $P_{1}$ in the Hausdorff metric (which, when combined with the obvious observation that any continuum can be approximated by a chainable one, gives that each continuum can be approximated by $P_{1}$ ). Furthermore, the homogeneity property characterizes $P_{1}$ among chainable continua [3].

The results in this section can be seen as dual to the above theory, much as the projective Fraïssé limit is dual to the Fraïssé limit. A chainable, hereditarily indecomposable continuum $P_{2}$ is constructed (Theorem 4.2). Two results are proved. First, $P_{2}$ is approximately projectively homogeneous (Theorem4.4(ii)) and, second, it is projectively universal among chainable continua (Theorem 4.4(i)). Moreover, 
the approximate projective homogeneity of $P_{2}$ characterizes it among chainable continua (Theorem 4.9).

The link between the Bing-Moise theory and its dual is provided by the old theorem of Bing [2] saying that up to a homeomorphism there is at most one chainable, hereditarily indecomposable continuum. Thus, $P_{1}$ and $P_{2}$ are homeomorphic.

2. It seems very likely that, using methods similar to the ones developed in the present paper, one will be able to prove existence, surjective universality, surjective homogeneity, and uniqueness of a universal pseudo-solenoid. This would extend the work of Rogers [12. In this context, it will be important to modify the notion of epimorphism and appropriately change the definitions of surjective universality and surjective homogeneity.

\section{REFERENCES}

1. R H Bing, A homogeneous indecomposable plane continuum, Duke Math. J. 15 (1948), 729742. MR0027144 (10:261a)

2. R H Bing, Concerning hereditarily indecomposable continua, Pacific J. Math. 1 (1951), 43-51. MR0043451(13265b)

3. R H Bing, Each homogeneous nondegenerate chainable continuum is a pseudo-arc, Proc. Amer. Math. Soc. 10 (1959), 345-346. MR0105072 (21:3818)

4. R. Engelking, General Topology, Sigma Series in Pure Mathematics, 6, Heldermann Verlag, Berlin, 1989. MR1039321 (91c:54001)

5. W. Hodges, Model Theory, Encyclopedia of Mathematics and its Applications, 42, Cambridge University Press, Cambridge, 1993. MR.1221741 (94e:03002)

6. G.R. Lehner, Extending homeomorphisms on the pseudo-arc, Trans. Amer. Math. Soc. 98 (1961), 369-394. MR0120608 (22:11358)

7. W. Lewis, Most maps of the pseudo-arc are homeomorphisms, Proc. Amer. Math. Soc. 91 (1984), 147-154. MR0735582 (85g:54025)

8. W. Lewis, The pseudo-arc, Bol. Soc. Mat. Mexicana (3) 5 (1999), 25-77. MR1692467 (2000f:54029)

9. J. Mioduszewski, A functional conception of snake-like continua, Fund. Math. 51 (1962/1963), 179-189. MR0144313 (26:1859)

10. E.E. Moise, A note on the pseudo-arc, Trans. Amer. Math. Soc. 67 (1949), 57-58. MR0033023 $(11: 382 \mathrm{~d})$

11. S.B. Nadler, Jr., Continuum Theory, Monographs and Textbooks in Pure and Applied Mathematics, 158, Marcel Dekker Inc., New York, 1992. MR1192552 (93m:54002)

12. J.T. Rogers, Jr., Pseudo-circles and universal circularly chainable continua, Illinois J. Math. 14 (1970), 222-237. MR0264622 (41:9213)

13. M. Smith, Every mapping of the pseudo-arc onto itself is a near homeomorphism, Proc. Amer. Math. Soc. 91 (1984), 163-166. MR0735585 (85i:54042)

Department of Mathematics, University of Illinois, 1409 W. Green St., Urbana, IlliNOIS 61801

Current address: Department of Mathematics, University of Louisville, Louisville, Kentucky 40292

E-mail address: tirwin@math.uiuc.edu

Department of Mathematics, University of Illinois, 1409 W. Green St., Urbana, IlliNOIS 61801

E-mail address: ssolecki@math.uiuc.edu 